\title{
Higgs production in association with a single top quark at the LHC
}

\author{
Federico Demartin $^{1}$, Fabio Maltoni ${ }^{1}$, Kentarou Mawatari ${ }^{2, a}$, Marco Zaro ${ }^{3,4}$ \\ ${ }^{1}$ Centre for Cosmology, Particle Physics and Phenomenology (CP3), Université catholique de Louvain, 1348 Louvain-la-Neuve, Belgium \\ 2 Theoretische Natuurkunde and IIHE/ELEM, Vrije Universiteit Brussel, and International Solvay Institutes, Pleinlaan 2, 1050 Brussels, Belgium \\ ${ }^{3}$ UMR 7589, LPTHE, Sorbonne Universités, UPMC Univ. Paris 06, 75005 Paris, France \\ ${ }^{4}$ UMR 7589, LPTHE, CNRS, 75005 Paris, France
}

Received: 14 April 2015 / Accepted: 19 May 2015 / Published online: 18 June 2015

(C) The Author(s) 2015. This article is published with open access at Springerlink.com

\begin{abstract}
We present a detailed study of Higgs boson production in association with a single top quark at the LHC, at next-to-leading order accuracy in QCD. We consider total and differential cross sections, at the parton level as well as by matching short distance events to parton showers, for both $t$-channel and $s$-channel production. We provide predictions relevant for the $\mathrm{LHC}$ at $13 \mathrm{TeV}$ together with a thorough evaluation of the residual uncertainties coming from scale variation, parton distributions, strong coupling constant and heavy quark masses. In addition, for $t$-channel production, we compare results as obtained in the 4-flavour and 5-flavour schemes, pinning down the most relevant differences between them. Finally, we study the sensitivity to a non-standard-model relative phase between the Higgs couplings to the top quark and to the weak bosons.
\end{abstract}

\section{Introduction}

The first Run of the LHC has already collected compelling evidence that the scalar particle observed at $125 \mathrm{GeV}$ is the one predicted by the Brout-Englert-Higgs symmetry breaking mechanism [1,2] of $S U(2)_{L} \times U(1)_{Y}$ as implemented in the Standard Model (SM) [3]. In such minimal case, the strengths of the Higgs boson couplings to the elementary particles, including the Higgs boson itself, are uniquely determined by their masses. While somewhat limited and subject to additional ad hoc assumptions, the first measurements of the Higgs couplings to fermions and vector bosons agree well with the SM predictions [4,5].

Such general agreement with the SM expectations and the absence of any evidence (from the LHC itself) of the existence of new states at the $\mathrm{TeV}$ scale, motivate a thorough study of the Higgs boson interactions at the Run II. In addition to the coupling strength determinations conducted so far,

\footnotetext{
a e-mail: kentarou.mawatari@vub.ac.be
}

the Lorentz structure of the vertices as well as the possible existence of a relative phase among the couplings need to be fully assessed. In order to gather the necessary information, the widest possible campaign of measurements has to be undertaken, including different production and decay modes of the Higgs boson. In addition, given the limited discriminating power of single channels, a global combination of the relevant measurements will be necessary. To achieve this goal at the LHC one needs to adopt a complete and consistent theoretical framework, able to encompass interactions that go beyond the SM (and possibly to organise them in terms of an ordering principle), and that allows the systematic inclusion of higher-order corrections, both QCD and electroweak (EW). This latter point is a conditio-sine-qua-non at the LHC, in order to control total rates and differential distributions and to estimate the residual uncertainties. Such a theoretical framework exists and amounts to "simply" extend the dimension-4 SM Lagrangian to all operators of higher dimensions (up to dimension- 6 in this first instance) consistent with the unbroken SM symmetries $S U(3)_{C} \times S U(2)_{L} \times U(1)_{Y}$; i.e. to consider the SM as an effective field theory valid up to a scale $\Lambda[6,7]$.

This work fits in the above general strategy and focuses on Higgs production in association with a single top quark. As in single top production, at the leading order (LO) in QCD one can organise the production mechanisms into three groups, based on the virtuality of the $W$ boson: $t$-channel production (Fig. 1), s-channel production (Fig. 2), and associated production with an on-shell $W$ boson. While characterised by a rather small cross section with respect to the main single Higgs production channels (gluon-gluon fusion, vector boson fusion and associated production, and $t \bar{t} H$ ), Higgs and single-top associated production features unique aspects that make this process particularly interesting for Higgs characterisation $[8,9]$. Notably, it is among the very few processes relevant for LHC phenomenology (together with $H \rightarrow \gamma \gamma$ and $g g \rightarrow Z H$ ) to be sensitive to the relative size and phase 

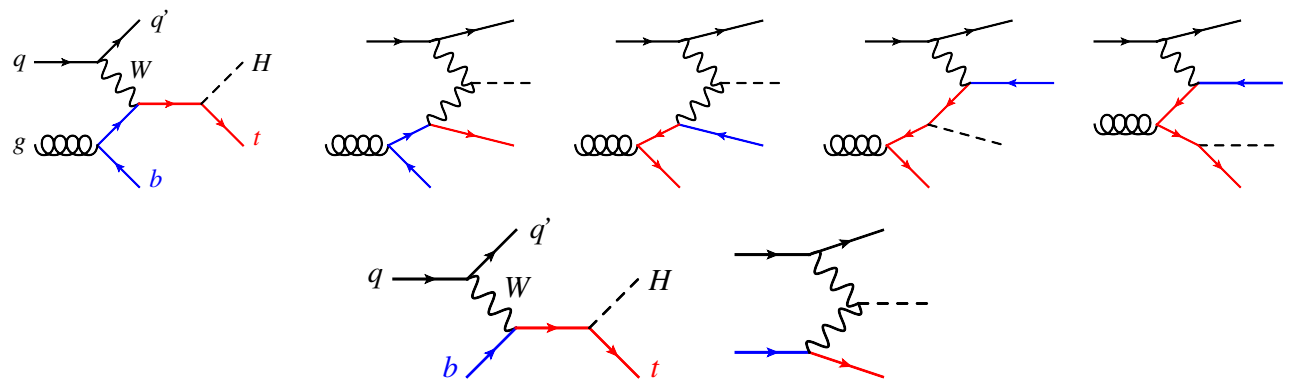

Fig. 1 LO Feynman diagrams for $t$-channel $t H$ production in the $4 \mathrm{~F}$ scheme (top) and in the $5 \mathrm{~F}$ scheme (bottom)
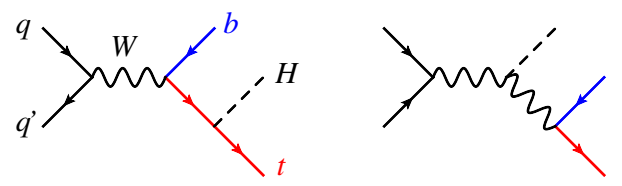

Fig. 2 LO Feynman diagrams for $s$-channel $t H$ production

of the coupling of the Higgs boson to the top quark and to the weak bosons. For $t$-channel and $W$-boson associated production, diagrams where the Higgs couples to the top quark interfere destructively with those where the Higgs couples to the $W$ boson (due to the unitarity of the weak interactions in the SM), making cross sections and distributions extremely sensitive to departures of the Higgs couplings from the SM predictions [10].

The aim of the first part of this work is to provide accurate SM predictions including QCD corrections at next-toleading order (NLO) for $t$ - and $s$-channel Higgs production in association with a single top quark, as well as reliable estimates for the residual uncertainties in rates and distributions. Particular attention is devoted to the uncertainty related to the different flavour schemes that can be adopted to compute the dominant $t$-channel production mode. The corresponding SM predictions are the necessary theoretical input to possibly assess the existence of deviations due to new physics (be it resonant or not); to this aim, the study of the uncertainties in total rates as well as in differential distributions becomes of primary importance.

We then consider how accurately and precisely the effects of the (only) dimension- 6 operator that modifies the value and the phase of the top quark Yukawa coupling can be predicted, again at the total as well as at the differential level. This information is useful to assess the reach of the LHC to constrain the relevance of this dimension-6 operator (i.e. to bound the complex coefficient in front) and, if deviations from the SM are detected, to quantify them.

The paper is organised as follows. In Sect. 2 we introduce the main features of the Higgs and top quark associated production. In Sect. 3 we focus on the $t$-channel production mode, with a special attention to the issues connected to the 4-flavour (4F) and 5-flavour (5F) schemes. We describe the settings of the calculation, present results in the SM for total rates up to NLO in QCD and their uncertainties, and finally show relevant differential distributions at NLO matched to a parton shower. In Sect. 4 we shortly consider the $s$-channel production mechanism, which has a much smaller impact on Higgs phenomenology in the SM. We evaluate the total cross sections and its uncertainties in the SM and show some representative distributions in comparison with the corresponding $t$-channel ones. In Sect. 5 we study the impact of an anomalous, CP-violating top quark Yukawa interaction on $t$-channel production, both at the total and differential cross section level. We summarise our findings in Sect. 6.

\section{Main features}

In this section we introduce the main features of Higgs production in association with a single top quark. As already mentioned in the introduction, at LO in QCD one can effectively organise the various production mechanisms into three groups, based on the virtuality of the $W$ boson: $t$-channel production features a space-like $W, s$-channel production a time-like $W$, and $W$-associated production an on-shell $W$ boson. One has to bear in mind that while this classification is certainly useful, it is not physical, being an approximation that holds only at LO and in the 5-flavour scheme. At higher orders in QCD, or using a different flavour scheme to define the processes, the separation becomes increasingly fuzzy, as it will be clarified at the end of this section.

As in single top production in the $\mathrm{SM}, t H$ production is always mediated by a $t W b$ vertex and therefore it entails the presence of a bottom quark either in the initial ( $t$-channel and $W$-associated) or in the final state ( $s$-channel). In the case of initial-state bottom quarks, two different approaches, the so-called 4F and 5F schemes, can be followed to perform perturbative calculations.

In the $4 \mathrm{~F}$ scheme one assumes that the typical scale of the hard process $\mathcal{Q}$ is not significantly higher than bottom quark mass, which in turn is considerably heavier than $\Lambda_{\mathrm{QCD}}$, $\mathcal{Q} \gtrsim m_{\mathrm{b}} \gg \Lambda_{\mathrm{QCD}}$. Technically, one constructs an effective 
theory of QCD with only four light flavours, where heavier quarks (bottom and top), being massive, do not contribute to the initial-state proton wave-function (in terms of parton distribution functions (PDFs)), nor to the running of the strong coupling, and they appear only as final-state particles. In so doing, mass effects in the kinematics of heavy-quark production are correctly taken into account already at the lowest order in perturbation theory. In addition, the matching to parton-shower programs is straightforward, the heavyquark mass acting as an infrared cutoff for inclusive observables. However, limitations might arise when $\mathcal{Q} \gg m_{\mathrm{b}}$ and one probes kinematic configurations which are dominated by almost collinear $g \rightarrow b \bar{b}$ splittings: in this case the accuracy of predictions can be spoiled by large logarithms $\log \left(\mathcal{Q}^{2} / m_{\mathrm{b}}^{2}\right)$ appearing at all orders in perturbative QCD. Were this the case, such large logarithms would harm the behaviour of a fixed order expansion in $\alpha_{s}$.

This issue can be addressed in the $5 \mathrm{~F}$ scheme (and improvements thereof), whose aim is to reorganise the perturbative expansion by resumming such logarithms via the DGLAP equations. One starts by assuming $\mathcal{Q} \gg m_{\mathrm{b}}$ and defines a scheme where power corrections of order $m_{\mathrm{b}} / \mathcal{Q}$ appear at higher orders in the $\alpha_{s}$ expansion. In practice, one sets the bottom mass to zero and includes bottom quarks in the initial state as proton constituents. ${ }^{1}$ In so doing, towers of logarithms associated with the initial-state $g \rightarrow b \bar{b}$ splitting are resummed to all orders in perturbation theory by evolving the perturbative bottom quark PDF via the DGLAP equations.

Computations in the $5 \mathrm{~F}$ scheme are typically much simpler than the corresponding $4 \mathrm{~F}$ ones, because of the lesser final-state multiplicity and the simpler phase space. This is for example the reason why single-top production is known at NNLO in the 5F scheme [12] while only at NLO in the $4 \mathrm{~F}$ [13]. For a systematic investigation of the sources of differences between the $4 \mathrm{~F}$ and $5 \mathrm{~F}$ schemes in single $b$-quark and double $b$-quark induced processes we refer the reader to $[14,15]$, respectively. In short, the $4 \mathrm{~F}$ and $5 \mathrm{~F}$ schemes differ in what kind of terms are pushed into the missing higherorder corrections. Therefore, as the accuracy of the predictions for a given observable increases, milder differences should be expected between the schemes. This provides a strong motivation to go at least to NLO accuracy in the computation of the $t$-channel cross section, in order to reduce the flavour-scheme dependence of the predictions and thus the overall theoretical uncertainty. The final accuracy, however, will depend on the specific observable considered, whose perturbative accuracy can be different in the two schemes.

\footnotetext{
1 The bottom mass can be reinstated explicitly at higher-orders by systematically including it in diagrams that do not feature bottom quarks in the initial state, the so-called S-ACOT scheme [11]. In this work we adopt a "pure" $5 \mathrm{~F}$ scheme where $m_{\mathrm{b}}=0$ throughout.
}

In the case of (Higgs and) single top production at hadron colliders, the $5 \mathrm{~F}$ scheme has also the operational advantage that allows an easy separation of the various production mechanisms into the three groups mentioned above. In the $5 \mathrm{~F}$ scheme the $t$-channel, $s$-channel and $W$-associated production are independent up to NLO and start to interfere only at NNLO, and the $W$-associated production interferes with $t \bar{t} H$ starting from NLO. In the 4F, on the other hand, the $t$ channel at NLO can interfere with the $s$-channel (at NNLO) and with $W$-associated production (if the $W$ decays hadronically), and the $W$-associated production also interferes with $t \bar{t} H$ already at the tree level. While the former interferences are very small and can be safely neglected if the aim is to evaluate the dominant $t$-channel cross section, the interference of $W$-associated production with $t \bar{t} H$ turns out instead to be quite large. The on-shell $W$ associated production therefore needs a dedicated study that we defer to a separate work.

\section{$3 t$-channel production}

In this section we present the SM predictions for $t$-channel Higgs plus single top production at the LHC (see Fig. 1), at NLO accuracy in QCD. We first describe the technical setup we have used for NLO simulations, the input parameters as well as the various sources of theoretical uncertainties. We then show results for the inclusive cross section at the LHC with $\sqrt{s}=13 \mathrm{TeV}$, discussing how to combine the theoretical uncertainties, and finally present NLO distributions matched to parton shower.

\subsection{NLO simulations, parameters and uncertainties}

In this work, we employ the MADGRAPH5_AMC@NLO framework [16], which allows to compute both inclusive cross sections and differential distributions matched to parton-shower programs, up to NLO accuracy in QCD, in a fully automatic way [17-20] once the relevant Feynman rules and $\mathrm{UV} / R_{2}$ counterterms for a given theory are provided in the form of a UFO model [21-23]. While these extra Feynman rules are available in MADGRAPH5_AMC@NLO by default for the SM, non-SM interactions that will be considered later in Sect. 5 are encoded in the HC_NLO_X0 model [24-26], publicly available online in the FEYNRULES repository [27].

In MADGRAPH5_AMC@NLO the code and events for $t$ channel $t H$ production at hadron colliders in the $4 \mathrm{~F}$ scheme can be automatically generated by issuing the following commands:

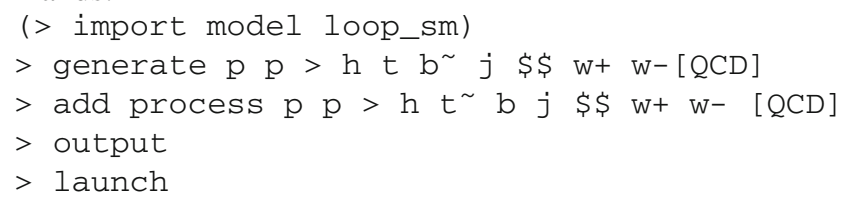


while the corresponding commands in the $5 \mathrm{~F}$ scheme are:

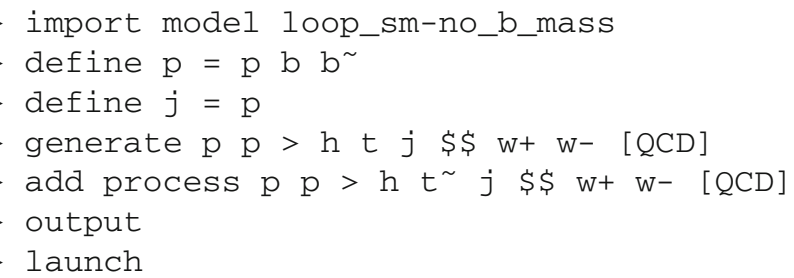

Note that the $\$ \$ \mathrm{w}^{+} \mathrm{w}-\operatorname{syntax}$ removes $s$-channel $t H$ diagrams as well as real-correction diagrams where an on-shell $W$ decays to two light quarks, which belong to $W$-associated production. The top quark decays are subsequently performed starting from the event file (in the Les Houches format [28]) by MADSPIN [29], following a procedure [30] that keeps spin correlations.

In the numerical calculation, the mass of the Higgs boson is set to $m_{\mathrm{H}}=125.0 \mathrm{GeV}$, while the mass of the top quark is set to $m_{\mathrm{t}}=173.3 \mathrm{GeV}$. We renormalise the top quark Yukawa coupling on-shell, setting it to $y_{t} / \sqrt{2}=m_{\mathrm{t}} / v$, where $v \sim 246 \mathrm{GeV}$ is the $\mathrm{EW}$ vacuum expectation value.

The on-shell mass of the bottom quark is set to

$m_{\mathrm{b}}=4.75 \pm 0.25 \mathrm{GeV}$,

where we take the uncertainty to be of $\mathcal{O}\left(\Lambda_{\mathrm{QCD}}\right)$, accordingly to the prescription in Ref. [31]. On the other hand, we set the bottom quark Yukawa coupling to zero, because effects related to the $H b \bar{b}$ interactions are negligible for this process. We remind that in the $4 \mathrm{~F}$ scheme the value of $m_{\mathrm{b}}$ enters the hard-scattering matrix element and the final-state phase space, while in the $5 \mathrm{~F}$ scheme it affects only the parton luminosity.

PDFs are evaluated by using three global fits: NNPDF2.3 [32], MSTW2008 [33] and CT10 [34], through the LHAPDF interface [35]. PDF uncertainties are computed for each PDF set, following the recipes summarised in [36]. A comparison among these three global fits allows to estimate the PDF systematic uncertainties related to the technical details of the fitting procedure employed by each group. We note that the above three PDF collaborations provide NLO PDF sets both in the $4 \mathrm{~F}$ and $5 \mathrm{~F}$ schemes, while only MSTW gives LO PDFs in both the schemes.

The reference value for the strong coupling constant we employ here is

$\alpha_{s}^{(\mathrm{NLO})}\left(m_{\mathrm{Z}}\right)=0.1190 \pm 0.0012$,

where the uncertainty is taken accordingly to the PDF4LHC recommendation [36,37], and the central value is chosen such that our $68 \%$ confidence interval encompasses the current PDG world average [38] and the best $\alpha_{s}\left(m_{\mathrm{Z}}\right)$ estimates obtained by each of the three PDF global fits [39-41]. We remark that the value in Eq. (2) is consistent with the $5 \mathrm{~F}$ description. Since the difference between $4 \mathrm{~F}$ and $5 \mathrm{~F}$ in the $\alpha_{s}$ running is limited to scales above $m_{\mathrm{b}}$, Eq. (2) can be translated into the following condition on $\alpha_{S}\left(m_{\mathrm{b}}\right)$ (running $\alpha_{s}$ at 2-loop accuracy)

$\alpha_{s}^{(\mathrm{NLO})}\left(m_{\mathrm{b}}\right)=0.2189 \pm 0.0042$,

which is now flavour-scheme independent.

CT10 does not provide PDF sets to compute $m_{\mathrm{b}}$ uncertainties in the $5 \mathrm{~F}$ scheme and PDF uncertainties in the 4F scheme; both CT10 and MSTW2008 do not provide 4F PDF sets with different $\alpha_{s}\left(m_{\mathrm{Z}}\right)$ values. Thus, it is possible to address all the various sources of uncertainty in both schemes only when using NNPDF2.3 parton distributions, while MSTW2008 and CT10 uncertainty bands can be sometimes underestimated (though just slightly, as we will see later in Sect. 3.2).

For matching short-distance events to parton shower we use the MC@NLO method [17] with PYTHIA8 [42], while HERWIG6 [43] has been used for a few comparisons. We recall that matching to PYTHIA6 [44] (virtualityordered, or $p_{T}$-ordered for processes with no final-state radiation) and HERWIG++ [45] are also available inside MADGRAPH5_AMC@NLO. Jets are reconstructed by means of the anti- $k_{T}$ algorithm [46] as implemented in FASTJET [47], with distance parameter $R=0.4$, and required to have

$p_{T}(j)>30 \mathrm{GeV}, \quad|\eta(j)|<4.5$.

A jet is identified as $b$-jet if a $b$-hadron (or $b$-quark for fixedorder calculations) is found among its constituents, and if the jet satisfies

$p_{T}\left(j_{b}\right)>30 \mathrm{GeV}, \quad\left|\eta\left(j_{b}\right)\right|<2.5$.

We assume $100 \% b$-tagging efficiency in this work.

\subsection{Total rates}

In this section we present the total cross section for $t$-channel production of a Higgs boson together with a single top quark (or antiquark), at NLO in QCD. The main sources of theoretical uncertainty that we address here are:

- renormalisation and factorisation scale dependence,

- $4 \mathrm{~F}$ and $5 \mathrm{~F}$ scheme dependence,

- PDF uncertainty,

$-\alpha_{s}\left(m_{\mathrm{Z}}\right)$ uncertainty,

$-m_{\mathrm{b}}$ uncertainty.

At the end of this section we will also briefly comment on the impact of the bottom quark Yukawa coupling and of the 


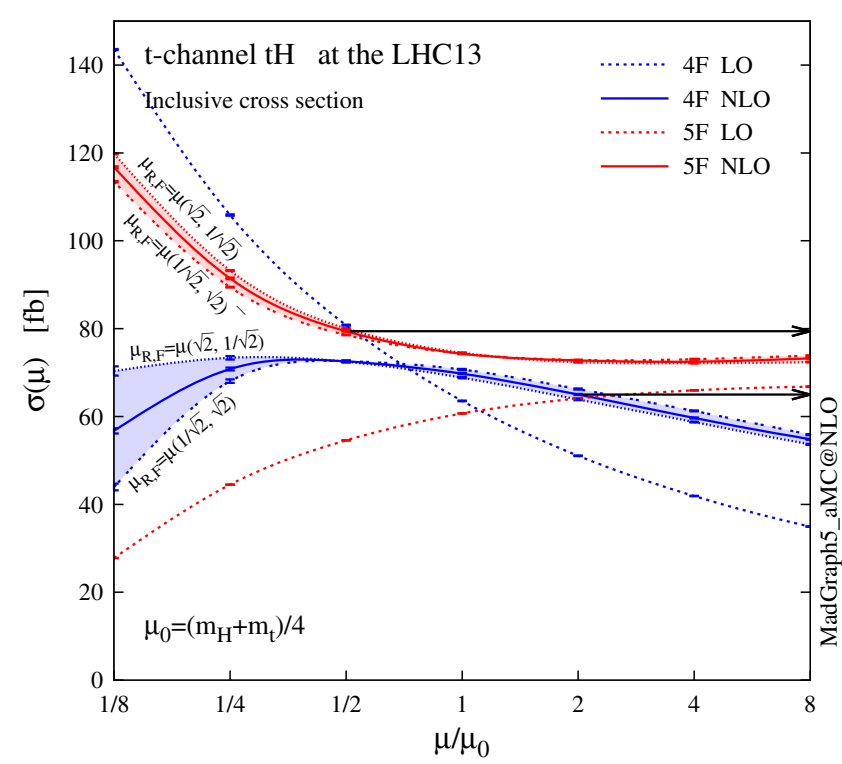

Fig. 3 Scale dependence of the total cross sections for the $p p \rightarrow$ $t H q+\bar{t} H q$ production at the $13-\mathrm{TeV}$ LHC, where the $4 \mathrm{~F}$ (blue) and $5 \mathrm{~F}$ (red) schemes are compared. LO (dashed) and NLO (solid) predictions with MSTW2008 LO/NLO PDFs are presented for $\mu_{R}=\mu_{F} \equiv \mu$, with a static (left figure) and a dynamic (right figure) scale choice. Two

dependence of the results on the Higgs and the top quark masses.

We start by showing in Fig. 3 the renormalisation and factorisation scale dependence of the LO and NLO total cross sections, both in the $4 \mathrm{~F}$ and $5 \mathrm{~F}$ schemes. We compute cross sections with two different scale choices, and vary $\mu_{R}=$ $\mu_{F} \equiv \mu$ around a central scale $\mu_{0}$ which is chosen as

$\mu_{0}^{s}=\left(m_{\mathrm{H}}+m_{\mathrm{t}}\right) / 4$

for the static scale choice (left figure), and

$\mu_{0}^{d}=H_{T} / 6=\sum_{i=H, t, b} m_{\mathrm{T}}(i) / 6$

for the event-by-event dynamic choice (right figure), where $m_{\mathrm{T}} \equiv \sqrt{m^{2}+p_{T}^{2}}$ is the transverse mass of a particle.

We find a pattern similar to the case of the single top production (see Fig. 3 in [13]). At LO the scale dependence in the $4 \mathrm{~F}$ scheme is stronger than in the $5 \mathrm{~F}$, simply because the $4 \mathrm{~F}$ calculation starts already at order $\alpha_{s}$. As expected, predictions at NLO are much more stable under the scale variation than at LO. We find that the $4 \mathrm{~F}$ and $5 \mathrm{~F}$ predictions are in better agreement if $\mu$ is chosen to be roughly a factor 4 (6) smaller than the typical hard scale of the process $m_{\mathrm{H}}+m_{\mathrm{t}}$ $\left(H_{T}\right)$ for the static (dynamic) scale choice. This is a known and general feature of $b$-initiated processes at hadron colliders [14]. At such reduced scales the $4 \mathrm{~F}$ and $5 \mathrm{~F}$ predictions are typically in good agreement, and this is indeed what we

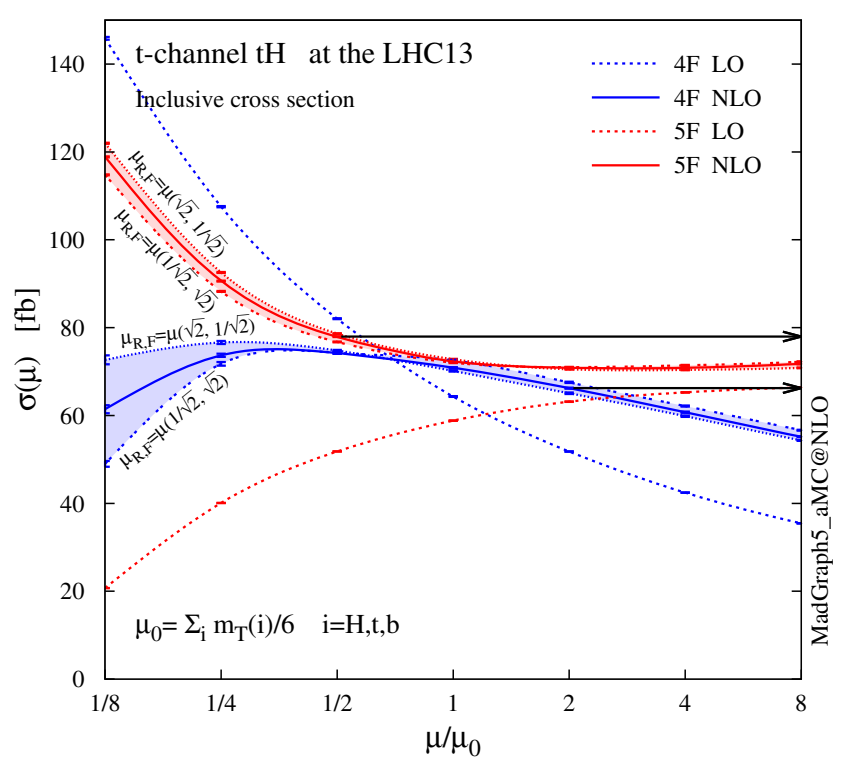

off-diagonal profiles of the scale dependence at NLO are also shown, for $\left(\mu_{R}=\sqrt{2} \mu, \mu_{F}=\mu / \sqrt{2}\right)$ and for $\left(\mu_{R}=\mu / \sqrt{2}, \mu_{F}=\sqrt{2} \mu\right)$. The black arrows visualise the envelope of the combined scale and flavour-scheme uncertainty defined in Eq. (8)

observe taking the reference scale choice $\mu_{0}$ as in Eqs. (6) and (7). Table 1 shows the corresponding values of the LO and NLO cross sections in Fig. 3, where the uncertainty from missing higher orders is estimated varying the scale $\mu$ by a factor 2 around $\mu_{0}$.

In Fig. 3 we also plot two off-diagonal $\left(\mu_{R} \neq \mu_{F}\right)$ slices of the NLO cross section surface in the plane $\left(\mu_{R}, \mu_{F}\right)$, shifted by a factor $\sqrt{2}$ in the direction orthogonal to the diagonal. The effects of off-diagonal scale choices are more pronounced in the $4 \mathrm{~F}$ scheme than in the $5 \mathrm{~F}$, even though in general they are quite modest, except at very low scales, i.e. comparable to $m_{\mathrm{b}}$. We conclude that, for our choice of $\mu_{0}$, the diagonal $\mu_{R}=\mu_{F}$ is sufficiently representative of the scale dependence of the total cross section, when the scale is varied by the usual factor two. We also observe that the scale value which minimises the flavour-scheme dependence is rather stable under shifts away from the diagonal.

We note that the scale dependence pattern is strongly correlated to the flavour scheme employed. Therefore, after we estimate the scale dependence of both $4 \mathrm{~F}$ and $5 \mathrm{~F}$ results (varying the scale $\mu_{F}=\mu_{R} \equiv \mu$ by a factor 2 around $\mu_{0}$ ), we define a combined scale and flavour-scheme uncertainty band by taking the envelope of the extremal points (shown by the black arrows in Fig. 3), and the best prediction for the cross section as the central point of this envelope. The total cross section at NLO and its combined scale plus flavour-scheme uncertainty are defined by

$\sigma_{\mathrm{NLO}}=\left(\sigma^{+}+\sigma^{-}\right) / 2, \quad \delta_{\mu+\mathrm{FS}}=\left(\sigma^{+}-\sigma^{-}\right) / 2$, 
Table 1 LO and NLO cross sections and corresponding $K$ factors for $t$-channel $t H$ production at the $13-\mathrm{TeV}$ LHC in the $4 \mathrm{~F}$ and $5 \mathrm{~F}$ schemes. MSTW2008 PDFs have been used. The integration error in the last digit(s) and the scale dependence by a factor 2 around the static and dynamic scale choices in Eqs. (6) and (7) are also reported

\begin{tabular}{llll}
\hline Scheme & $\sigma_{\mathrm{LO}}[\mathrm{fb}]$ & $\sigma_{\mathrm{NLO}}[\mathrm{fb}]$ & $K$ \\
\hline $4 \mathrm{~F}\left(\mu_{0}^{s}\right)$ & $63.46(8)_{-19.7 \%}^{+27.2 \%}$ & $69.43(7)_{-5.8 \%}^{+4.0 \%}$ & 1.09 \\
$5 \mathrm{~F}\left(\mu_{0}^{s}\right)$ & $60.66(6)_{-10.0 \%}^{+5.6 \%}$ & $73.45(8)_{-2.3 \%}^{+7.0 \%}$ & 1.21 \\
$4 \mathrm{~F}\left(\mu_{0}^{d}\right)$ & $64.31(8)_{-19 \%}^{+27.6 \%}$ & $71.29(10)_{-7.1 \%}^{+3.8 \%}$ & 1.11 \\
$5 \mathrm{~F}\left(\mu_{0}^{d}\right)$ & $58.83(5)_{-11.9 \%}^{+7.6 \%}$ & $71.54(7)_{-2.1 \%}^{+7.3 \%}$ & 1.22 \\
\hline
\end{tabular}

Table 2 NLO cross sections and uncertainties for $p p \rightarrow t H q, \bar{t} H q$ and $(t H q+\bar{t} H q)$ at the 13-TeV LHC. NNPDF2.3 PDFs have been used (NNPDF2.1 for $m_{\mathrm{b}}$ uncertainty in 5F). The integration uncertainty in the last digit(s) (in parentheses) as well as the scale dependence and the combined PDF $+\alpha_{s}+m_{\mathrm{b}}$ uncertainty in Eq. (11) (in \%) are reported. The individual PDF, $\alpha_{s}$ and $m_{\mathrm{b}}$ uncertainties are also presented as a reference

\begin{tabular}{|c|c|c|c|c|c|c|c|c|c|c|c|c|c|}
\hline & $t$-channel & $\sigma_{\mathrm{NLO}}^{\left(\mu_{0}^{s}\right)}[\mathrm{fb}]$ & $\delta_{\mu}^{\%}$ & $\delta_{\mathrm{PDF}+\alpha_{s}+m_{\mathrm{b}}}^{\%}$ & $\delta_{\mathrm{PDF}}^{\%}$ & $\delta_{\alpha_{s}}^{\%}$ & $\delta_{m_{\mathrm{b}}}^{\%}$ & $\sigma_{\mathrm{NLO}}^{\left(\mu_{0}^{d}\right)}[\mathrm{fb}]$ & $\delta_{\mu}^{\%}$ & $\delta_{\mathrm{PDF}+\alpha_{s}+m_{\mathrm{b}}}^{\%}$ & $\delta_{\mathrm{PDF}}^{\%}$ & $\delta_{\alpha_{s}}^{\%}$ & $\delta_{m_{\mathrm{b}}}^{\%}$ \\
\hline \multirow[t]{3}{*}{$4 \mathrm{~F}$} & $t H$ & $45.90(7)$ & $\begin{array}{l}+3.6 \\
-6.3\end{array}$ & $\begin{array}{l}+2.3 \\
-2.3\end{array}$ & \pm 0.9 & $\begin{array}{l}+0.6 \\
-0.9\end{array}$ & $\begin{array}{l}+2.0 \\
-2.0\end{array}$ & $46.67(8)$ & $\begin{array}{l}+4.3 \\
-6.1\end{array}$ & $\begin{array}{l}+3.2 \\
-1.9\end{array}$ & \pm 0.9 & $\begin{array}{l}+1.6 \\
{ }_{-0.4}\end{array}$ & $\begin{array}{l}+2.6 \\
-1.6\end{array}$ \\
\hline & $\bar{t} H$ & 23.92(3) & $\begin{array}{l}+4.2 \\
-6.6\end{array}$ & $\begin{array}{l}+2.5 \\
-2.7\end{array}$ & \pm 1.4 & $\begin{array}{l}+1.6 \\
-1.8\end{array}$ & $\begin{array}{l}+1.4 \\
-1.5\end{array}$ & $24.47(5)$ & $\begin{array}{l}+4.4 \\
-6.8\end{array}$ & $\begin{array}{l}+2.5 \\
-2.3\end{array}$ & \pm 1.4 & $\begin{array}{l}+1.4 \\
-1.4\end{array}$ & $\begin{array}{l}+1.6 \\
-1.2\end{array}$ \\
\hline & $t H+\bar{t} H$ & 69.81(11) & $\begin{array}{l}+3.2 \\
-6.6\end{array}$ & $\begin{array}{l}+2.8 \\
-2.5\end{array}$ & \pm 0.9 & $\begin{array}{l}+1.6 \\
-1.7\end{array}$ & $\begin{array}{l}+2.1 \\
-1.6\end{array}$ & $71.20(11)$ & $\begin{array}{l}+4.3 \\
-6.5\end{array}$ & $\begin{array}{l}+3.0 \\
-2.4\end{array}$ & \pm 0.9 & $\begin{array}{l}+2.0 \\
-1.1\end{array}$ & $\begin{array}{l}+2.0 \\
-1.9\end{array}$ \\
\hline \multirow[t]{3}{*}{$5 \mathrm{~F}$} & $t H$ & $48.80(5)$ & $\begin{array}{l}+7.1 \\
-1.7\end{array}$ & $\begin{array}{l}+2.8 \\
-2.3\end{array}$ & \pm 1.0 & $\begin{array}{l}+1.7 \\
-1.1\end{array}$ & $\begin{array}{l}+2.0 \\
-1.8\end{array}$ & $47.62(5)$ & $\begin{array}{l}+7.4 \\
-2.2\end{array}$ & $\begin{array}{l}+3.0 \\
-2.4\end{array}$ & \pm 1.0 & $\begin{array}{l}+1.6 \\
-0.8\end{array}$ & $\begin{array}{l}+2.4 \\
-2.0\end{array}$ \\
\hline & $\bar{t} H$ & $25.68(3)$ & $\begin{array}{l}+6.8 \\
-2.0\end{array}$ & $\begin{array}{l}+3.4 \\
-2.9\end{array}$ & \pm 1.4 & $\begin{array}{l}+1.9 \\
-1.5\end{array}$ & $\begin{array}{l}+2.5 \\
-2.0\end{array}$ & $25.07(3)$ & $\begin{array}{l}+7.4 \\
-2.1\end{array}$ & $\begin{array}{l}+3.2 \\
-2.9\end{array}$ & \pm 1.4 & $\begin{array}{l}+1.7 \\
-1.8\end{array}$ & $\begin{array}{l}+2.4 \\
-1.8\end{array}$ \\
\hline & $t H+\bar{t} H$ & $74.80(9)$ & $\begin{array}{l}+6.8 \\
-2.4\end{array}$ & $\begin{array}{l}+3.0 \\
-2.4\end{array}$ & \pm 1.0 & $\begin{array}{l}+1.5 \\
-1.1\end{array}$ & $\begin{array}{l}+2.4 \\
-1.9\end{array}$ & $72.79(7)$ & $\begin{array}{l}+7.4 \\
-2.4\end{array}$ & $\begin{array}{l}+2.9 \\
-2.3\end{array}$ & \pm 1.0 & $\begin{array}{l}+1.2 \\
-1.4\end{array}$ & $\begin{array}{l}+2.4 \\
{ }_{-1.6}\end{array}$ \\
\hline
\end{tabular}

where

$$
\begin{aligned}
& \sigma^{+}=\max _{\mu \in\left[\mu_{0} / 2,2 \mu_{0}\right]}\left\{\sigma_{\mathrm{NLO}}^{4 \mathrm{~F}}(\mu), \sigma_{\mathrm{NLO}}^{5 \mathrm{~F}}(\mu)\right\}, \\
& \sigma^{-}=\min _{\mu \in\left[\mu_{0} / 2,2 \mu_{0}\right]}\left\{\sigma_{\mathrm{NLO}}^{4 \mathrm{~F}}(\mu), \sigma_{\mathrm{NLO}}^{5 \mathrm{~F}}(\mu)\right\} .
\end{aligned}
$$

Now we turn to the PDF, $\alpha_{s}\left(m_{\mathrm{Z}}\right)$ and $m_{\mathrm{b}}$ uncertainties. In principle these three uncertainties can be correlated. However, the correlations are very small and can be often neglected in combinations. For example, using NNPDF, we have explicitly checked that the combined PDF $+\alpha_{s}$ uncertainty computed with full correlations differs from the one without correlations by $0.1 \%$ at most. In the $4 \mathrm{~F}$ scheme $m_{\mathrm{b}}$ is independent of PDF and $\alpha_{s}$, while we confirmed that the uncertainty correlation between PDF and $m_{\mathrm{b}}$ in the $5 \mathrm{~F}$ scheme is well below the percent level. Moreover, the correlation between $\alpha_{s}$ and $m_{\mathrm{b}}$ is tiny and can be neglected [31]. We note that neglecting correlations allows us to compare PDF uncertainty bands at a common $\alpha_{s}$ value, once central predictions (computed with this common $\alpha_{s}$ ) are dressed with their corresponding fractional PDF uncertainty (computed with each group's dedicated set). This is a known fact and it has been extensively used in recent PDF benchmarks [48].

Given that correlations among the uncertainties are very small, as discussed above, and also that not every PDF set allows to take into account all the correlations, we define the combined PDF, $\alpha_{s}$ and $m_{\mathrm{b}}$ uncertainty by simply summing the uncertainties in quadrature as

$\delta_{\mathrm{PDF}+\alpha_{s}+m_{\mathrm{b}}}^{ \pm}=\sqrt{\left(\delta_{\mathrm{PDF}}^{ \pm}\right)^{2}+\left(\delta_{\alpha_{s}}^{ \pm}\right)^{2}+\left(\delta_{m_{\mathrm{b}}}^{ \pm}\right)^{2}}$

Finally, we define the total theoretical uncertainty as the linear sum of the upper and lower variations for $\delta_{\mu}$ and $\delta \mathrm{PDF}+\alpha_{s}+m_{\mathrm{b}}$ in a given flavour scheme.

In Table 2, we report the NLO cross sections and their uncertainties at the 13-TeV LHC, for $t$-channel $t H$ and $\bar{t} H$ productions separately, and for their sum $t H+\bar{t} H$. Results are shown, using NNPDF2.3, in the 4F and 5F scheme for the static and dynamic scale choices in Eqs. (6) and (7), including the sources of uncertainty discussed above: scale uncertainty and combined PDF, $\alpha_{s}\left(m_{\mathrm{Z}}\right)$ and $m_{\mathrm{b}}$ one as well as the individual ones. The predictions in the combination of the $4 \mathrm{~F}$ and 5F schemes defined in Eq. (8) are presented in Table 3. The theoretical uncertainty is dominated by the combined scale and flavour-scheme uncertainty $\delta_{\mu+\mathrm{FS}}$ over the PDF, $\alpha_{s}$ and $m_{\mathrm{b}}$ uncertainty $\delta \mathrm{PDF}+\alpha_{s}+m_{\mathrm{b}}$. Figure 4 summarises the NLO cross sections and the theoretical uncertainties for $t$-channel $t H$ production, including the MSTW2008 and CT10 predictions.

We conclude this section by commenting on two additional minor sources of uncertainty. The first one is related to the value of the Higgs and top quark masses. In Table 4 we collect results for the $t$-channel NLO cross section (in the $5 \mathrm{~F}$ scheme only) with parametric variations of $1 \mathrm{GeV}$ in $m_{\mathrm{H}}$ and 
Table 3 Same as Table 2, but for the flavour-scheme combined results, according to Eq. (8)

\begin{tabular}{|c|c|c|c|c|c|c|c|}
\hline & $t$-channel & $\sigma_{\mathrm{NLO}}^{\left(\mu_{0}^{s}\right)}[\mathrm{fb}]$ & $\delta_{\mu+\mathrm{FS}}^{\%}$ & $\delta_{\mathrm{PDF}+\alpha_{s}+m_{\mathrm{b}}}^{\%}$ & $\sigma_{\mathrm{NLO}}^{\left(\mu_{0}^{d}\right)}[\mathrm{fb}]$ & $\delta_{\mu+\mathrm{FS}}^{\%}$ & $\delta_{\mathrm{PDF}+\alpha_{s}+m_{\mathrm{b}}}^{\%}$ \\
\hline \multirow[t]{3}{*}{$4 \mathrm{~F}+5 \mathrm{~F}$} & $t H$ & $47.64(7)$ & \pm 9.7 & $\begin{array}{l}+2.9 \\
-2.3\end{array}$ & $47.47(6)$ & \pm 7.7 & $\begin{array}{l}+3.1 \\
-1.8\end{array}$ \\
\hline & $\bar{t} H$ & $24.88(4)$ & \pm 10.2 & $\begin{array}{l}+3.5 \\
-2.6\end{array}$ & $24.86(3)$ & \pm 8.3 & $\begin{array}{l}+3.3 \\
-2.3\end{array}$ \\
\hline & $t H+\bar{t} H$ & $72.55(10)$ & \pm 10.1 & $\begin{array}{l}+3.1 \\
-2.4\end{array}$ & $72.37(10)$ & \pm 8.0 & $\begin{array}{l}+2.9 \\
-2.3\end{array}$ \\
\hline
\end{tabular}

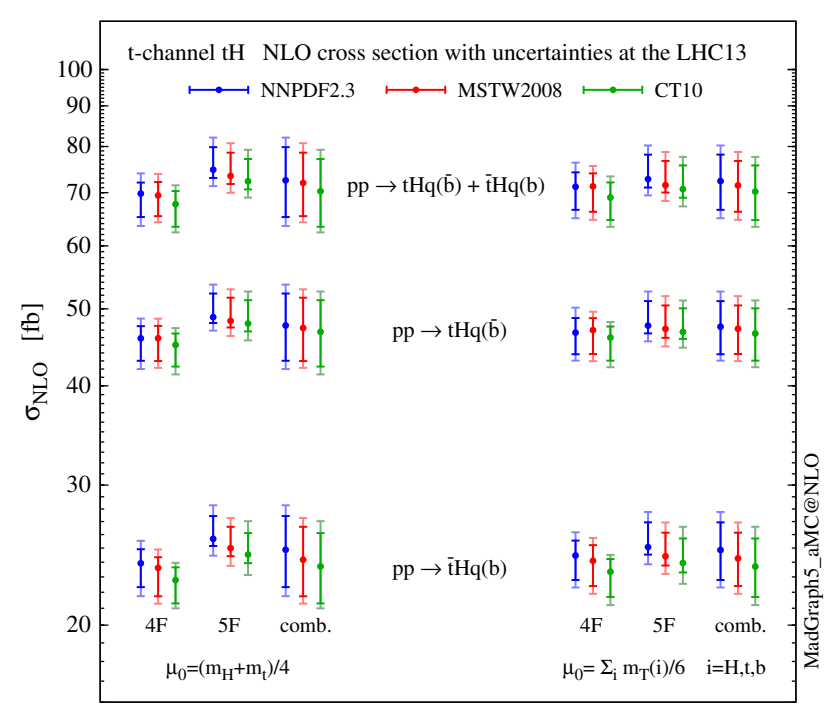

Fig. 4 Summary plot of the NLO cross sections with uncertainties for Higgs production associated with a single top quark, via a $t$-channel $W$ boson, at the 13-TeV LHC. For the uncertainties, the inner ticks display the scale (plus combined flavour-scheme) dependence $\delta_{\mu}(+\mathrm{FS})$, while the outer ones include $\delta_{\mathrm{PDF}+\alpha_{s}+m_{\mathrm{b}}}$

$m_{\mathrm{t}}$. The variations have a modest impact on the total cross section, about $1 \%$ only when both masses are varied in the same direction. From the combination of Tevatron and LHC experimental results [49] the top mass is currently known with a precision better than $1 \mathrm{GeV}$, while the combination of the latest ATLAS and CMS measurements of the Higgs mass gives a precision better than $0.5 \mathrm{GeV}$ [50]. We conclude that the impact of these uncertainties on the $t$-channel cross section at the LHC is below $1 \%$. The last source of uncertainty we discuss is the Yukawa coupling of the bottom quark. We have checked that it is completely negligible, both in the $4 \mathrm{~F}$

Table 4 Higgs and top quark mass dependence of the NLO cross sections in the 5F scheme for $p p \rightarrow t H q+\bar{t} H q$ at the LHC with $\sqrt{s}=$ $13 \mathrm{TeV}$. NNPDF2.3 PDFs have been used with $\mu_{0}=\left(m_{\mathrm{H}}+m_{\mathrm{t}}\right) / 4$. The and $5 \mathrm{~F}$ schemes, the impact of turning $y_{b}$ on/off at NLO being smaller than the numerical accuracy $(0.1-0.2 \%)$. Finally, we remind the reader that EW corrections for this process are presently unknown, and these could have an impact on the accuracy of the present predictions.

\subsection{Distributions}

We now present a selection of kinematical distributions for the combined $t$-channel $t H+\bar{t} H$ production at the $13-\mathrm{TeV}$ LHC, with NLO corrections and matching to a parton shower $(\mathrm{NLO}+\mathrm{PS})$. For the sake of brevity, we do not consider top and anti-top processes separately in this section, and will dub with $t$ both the top quark and its antiquark. Our main interest here is to assess the precision of the predictions for $t$-channel production, therefore we do not specify any decay mode for the Higgs boson, i.e. we leave it stable in the simulation. On the other hand, we consider (leptonic) top decays, which allows us to compare the distributions of $b$-jets coming from the hard scattering to the ones coming from the top quark.

For the kinematical distributions, we use NNPDF 2.3 PDFs and the PYTHIA8 parton shower. We have compared predictions obtained with the MSTW2008 and CT10 PDF sets and found no difference worth to report. We have also employed the HERWIG6 parton shower to verify that some important conclusions on the difference of the radiation pattern between $4 \mathrm{~F}$ and $5 \mathrm{~F}$ schemes were not dependent on shower programs. We estimate the scale dependence by varying $\mu_{R}$ and $\mu_{F}$ independently by a factor two around the reference dynamic scale $H_{T} / 6$ defined in Eq. (7), which provides smaller scale dependence than the static choice for differential distributions, especially for the high- $p_{T}$ region.

figures in parentheses are the $\%$ variations with respect to the reference cross section, computed with $m_{\mathrm{H}}=125.0 \mathrm{GeV}$ and $m_{\mathrm{t}}=173.3 \mathrm{GeV}$

\begin{tabular}{lllll}
\hline$\sigma_{\mathrm{NLO}}^{\left(5 \mathrm{~F} \mu_{0}^{s}\right)}[\mathrm{fb}]$ & & $m_{\mathrm{t}}[\mathrm{GeV}]$ & \\
\cline { 2 - 4 } & & 172.3 & 173.3 & 174.3 \\
\hline$m_{\mathrm{H}}[\mathrm{GeV}]$ & 124.0 & $75.54(+1.0 \%)$ & $75.18(+0.5 \%)$ & $74.99(+0.3 \%)$ \\
& 125.0 & $75.10(+0.4 \%)$ & 74.80 & $74.43(-0.5 \%)$ \\
\hline
\end{tabular}



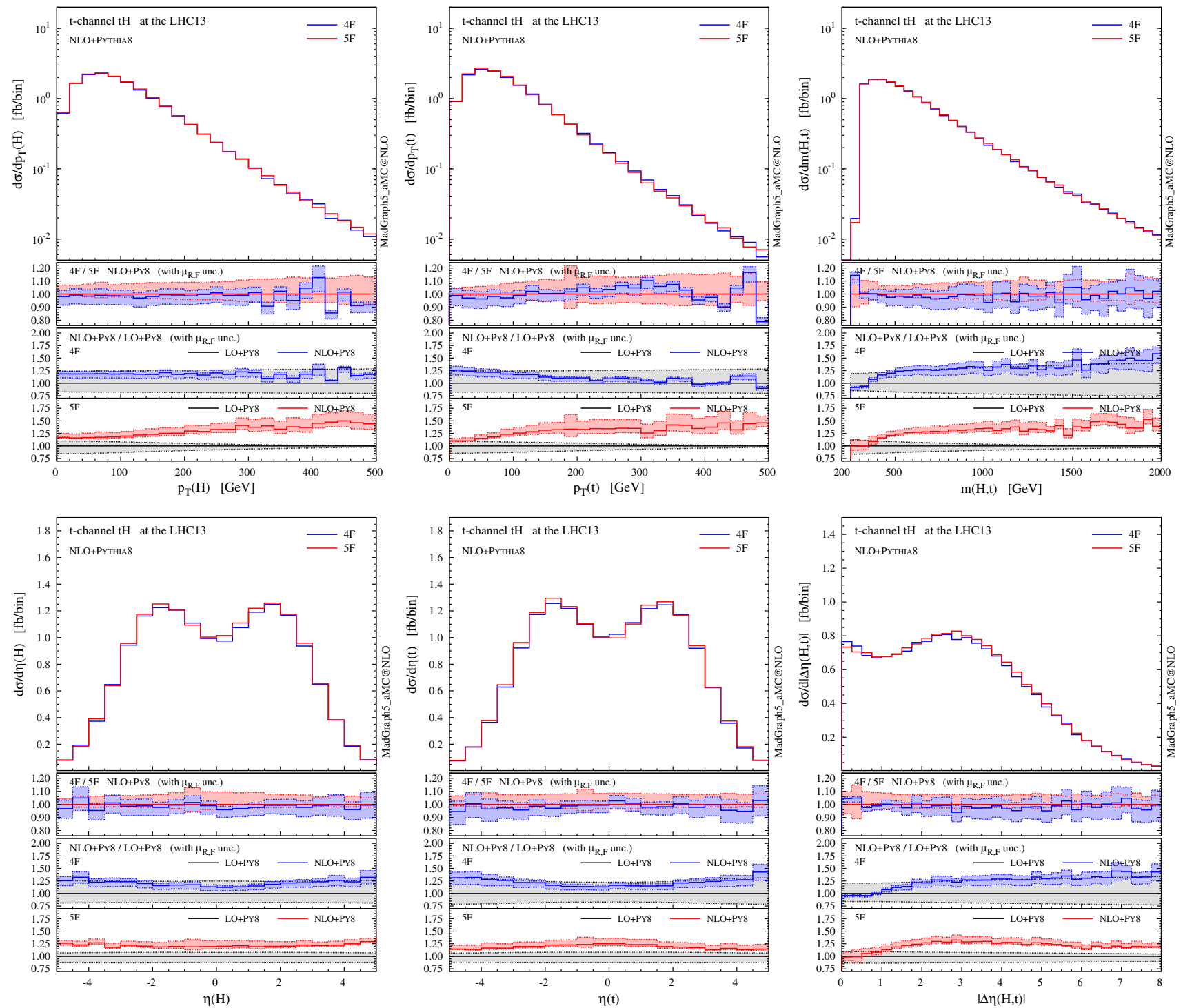

Fig. 5 Representative differential distributions for the Higgs boson and the top quark at NLO + PS accuracy in $t$-channel $t H$ associated production at the 13-TeV LHC. The lower panels provide information on the differences between $4 \mathrm{~F}$ and $5 \mathrm{~F}$ schemes as well as the differential $K$ factors in the two schemes

We start by showing in Fig. 5 differential distributions for the Higgs boson and the top quark (before they decay). The first observation is that NLO distributions in the $4 \mathrm{~F}$ and $5 \mathrm{~F}$ schemes are in excellent agreement within their respective uncertainty associated to scale variation, i.e. within the $10 \%$ level. Interestingly, though, differential $K$ factors (information in the insets below) are more pronounced for the $5 \mathrm{~F}$ than for the $4 \mathrm{~F}$ scheme, the NLO results in the $5 \mathrm{~F}$ scheme typically being out of the uncertainties as estimated from scale variation at LO. It should be noted that the LO process in the $5 \mathrm{~F}$ scheme does not depend on the renormalisation scale, and therefore its smaller uncertainty (especially in the high- $p_{T}$ region) can be an artefact of the scheme. Results in the $5 \mathrm{~F}$ tend to have a scale uncertainty that increases with $p_{T}$ much more than in the 4F, but in most cases the differences are not striking. Slightly larger deviations between $4 \mathrm{~F}$ and $5 \mathrm{~F}$ appear only very close to the $t H$ threshold, a region where we expect the $4 \mathrm{~F}$ scheme to catch the underlying physics already at LO.

In Fig. 6 we present distributions for the two hardest jets which are not tagged as $b$-jets. Jets and $b$-jets are defined in Eqs. (4) and (5). The contributions from the non-taggable forward $b$-jets $(2.5<|\eta|<4.5)$ are also denoted by shaded histograms as a reference. The jet with the highest transverse momentum $\left(j_{1}\right)$ tends to be produced in the forward region, very much like in single-top and VBF production. Most of the time this jet can be clearly associated to the light-quark current in the hard scattering. The very good agreement between 

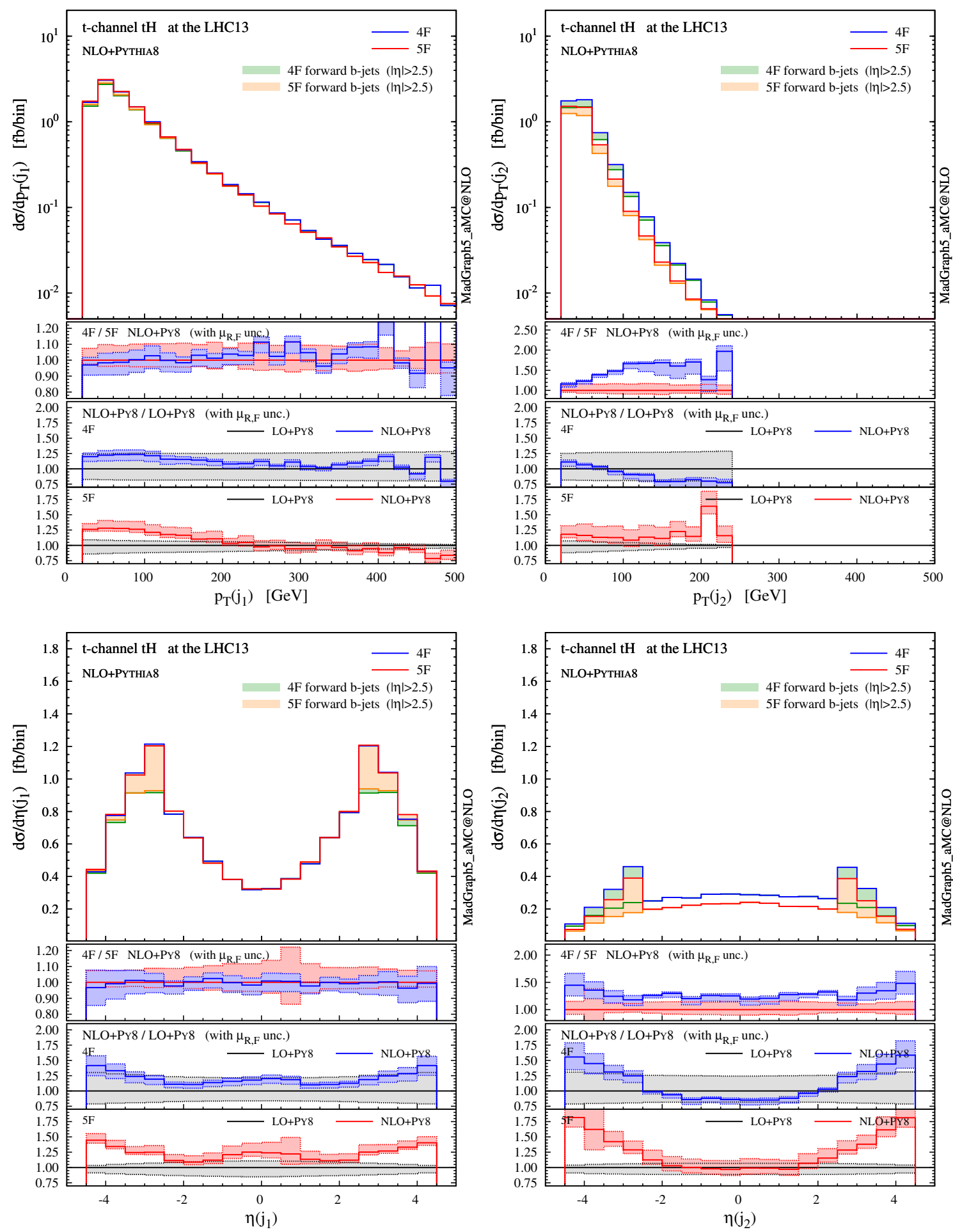

Fig. 6 Same as Fig. 5, but for the two hardest jets. The contributions from non-taggable forward b-jets are also shown by shaded histograms as a reference

$4 \mathrm{~F}$ and $5 \mathrm{~F}$ is manifest. This is expected as this observable should not be too sensitive on the details of heavy-quark current, as colour connections between the two currents are either vanishing or suppressed at the order in QCD we are working. On the other hand, sizeable differences arise for the second-hardest jet $\left(j_{2}\right)$, which shows a much steeper $p_{T}$ spectrum and tends to be produced centrally. The difference between predictions in the $4 \mathrm{~F}$ and $5 \mathrm{~F}$ schemes is often much larger than the scale uncertainty band (which is more pronounced in the $5 \mathrm{~F}$ scheme in the bulk of the events). We will 

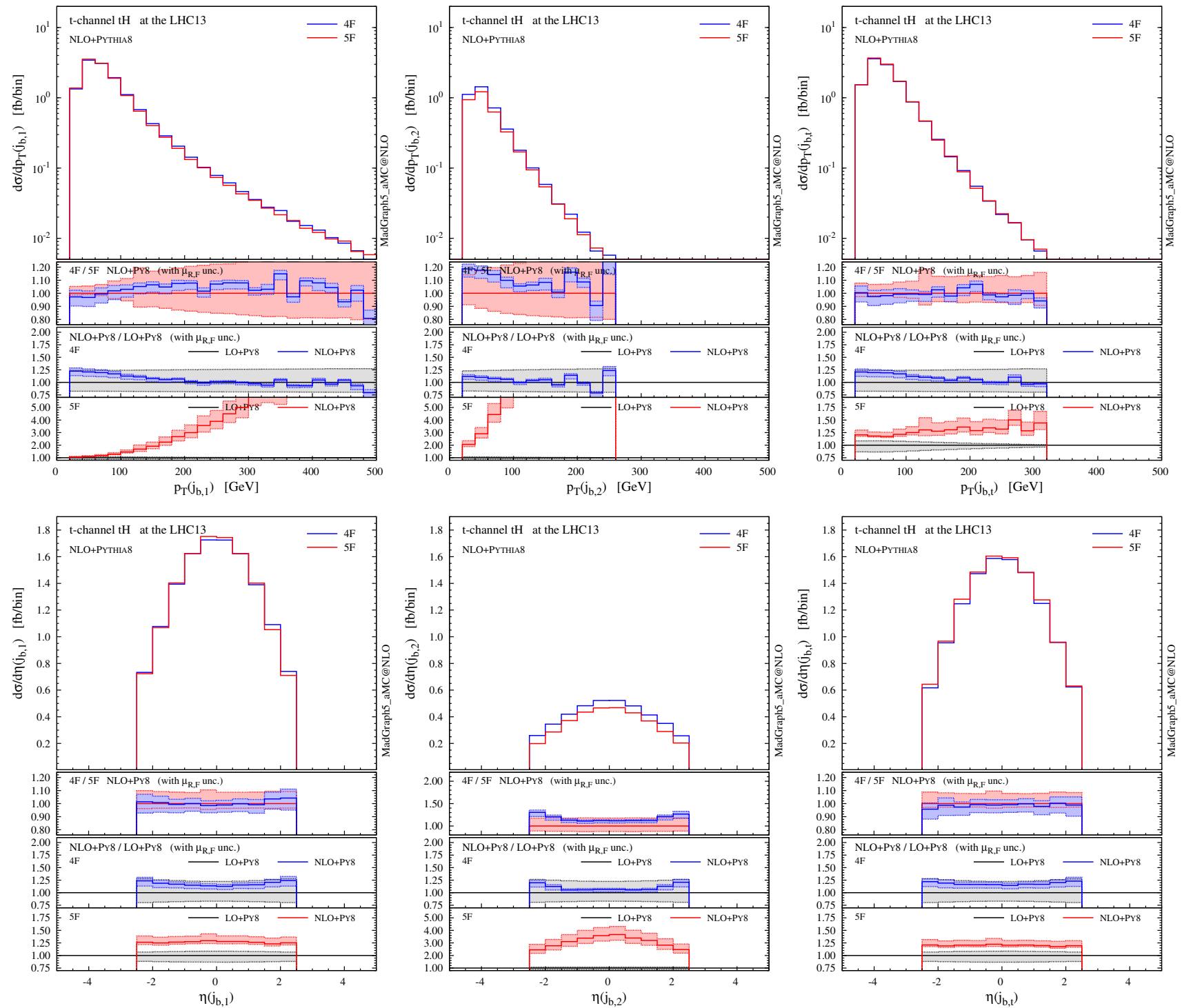

Fig. 7 Same as Fig. 5, but for the $b$-tagged jets. On the right column the distributions for the $b$-jet coming from the top quark decay, selected by using Monte Carlo information, are shown

discuss further this feature when presenting jet multiplicities in the following.

In Fig. 7 we show the analogous distributions for the $b$ tagged jets. These are all the jets containing a $b$-hadron and falling inside the acceptance of the tracking system, Eq. (5). We consider the two hardest $b$-jets $\left(j_{b, 1}\right.$ and $\left.j_{b, 2}\right)$ in the event regardless of their origin and, separately, we study the $b$-jet coming from the top quark decay $j_{b, t}$ (tagged by using Monte Carlo information). The $p_{T}$ spectrum of $j_{b, 1}$ has a rather long tail compared to $j_{b, 2}$ and, at variance with light jets, all the $b$-jets tend to be produced in the central region. Scale dependence at NLO is rather small in the $4 \mathrm{~F}$ scheme, never reaching $10 \%$ and being typically around $5 \%$. Differences between $4 \mathrm{~F}$ and $5 \mathrm{~F}$ predictions are visible, specially in the uncertainty band of $j_{b, 2}$ in the $5 \mathrm{~F}$ scheme; this is of course expected, given that this observable is described only at LO accuracy in this scheme. Quite remarkably, however, these differences at NLO are often significantly less pronounced than in the case of light jets (specially for the second jet), while naively one might expect the $b$-jet observables to be mostly affected by the flavour-scheme choice. On the other hand, at LO the inadequacy of the $5 \mathrm{~F}$ scheme to describe $b$-jets is evident.

Comparing the transverse momentum of $j_{b, t}$ (first row, right plot in Fig. 7) to the corresponding spectra of $j_{b, 1}$ and $j_{b, 2}$, it can be inferred that $b$-jets from the top quark mostly contribute to the hardest $b$-jet $(j b, 1)$ spectrum at low $p_{T}$. On the other hand, as the $p_{T}$ tail falls much more rapidly for $j_{b, t}$ than for $j_{b, 1}$, gluon splitting in the hard scattering is the predominant mechanism at high $p_{T}$, and thus the main source of $b$-jets in this region. This observation also explains why the scale dependence in the $5 \mathrm{~F}$ is small for low $p_{T}\left(j_{b, 1}\right)$, 


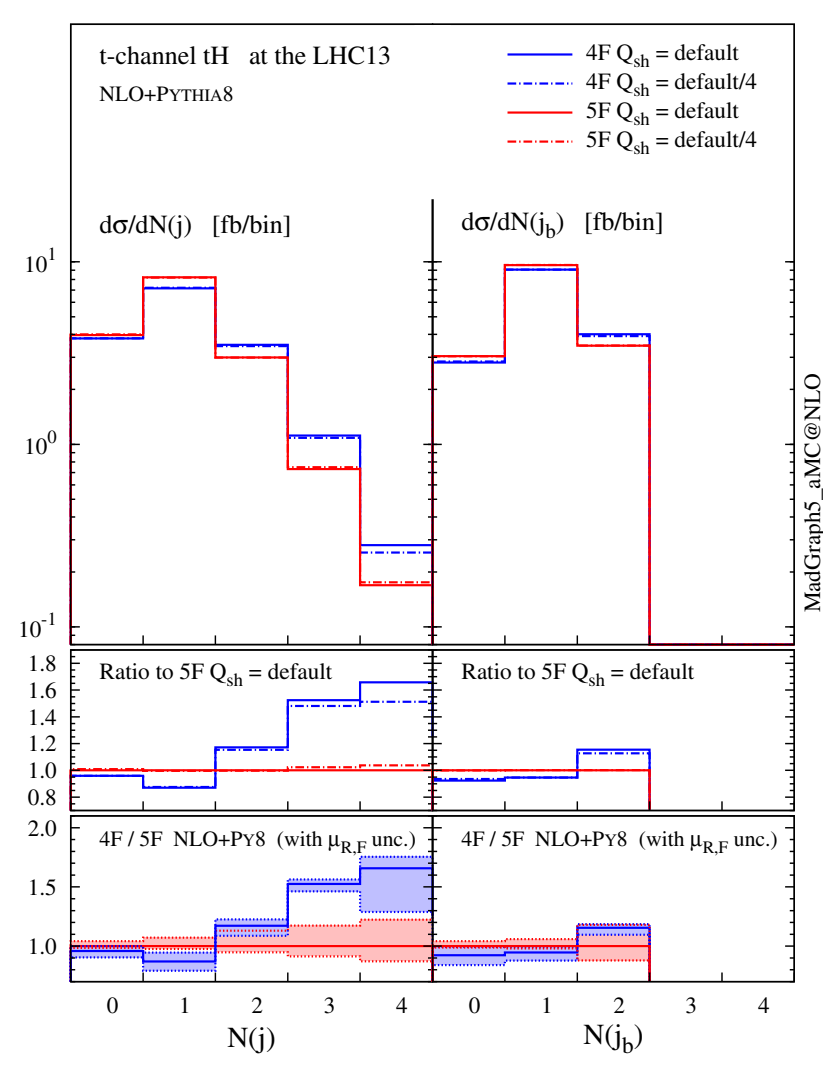

Fig. 8 Jet rates at NLO + PS accuracy in $4 \mathrm{~F}$ and $5 \mathrm{~F}$ schemes with different choices of the shower scales

which is described at NLO accuracy, and increases sharply in the high- $p_{T}\left(j_{b, 1}\right)$ region, where the physics is dominated by the transverse dynamics of the $g \rightarrow b \bar{b}$ splitting, which is described only at LO.

We conclude this section by studying the jet multiplicities, which are sensitive to the flavour scheme as well as to the choice of the shower scale. As argued in [14], the dynamics of $g \rightarrow b \bar{b}$ splitting takes place at a scale which is typically lower than the hard scale of the process $m_{\mathrm{t}}+m_{\mathrm{H}}$ or $H_{T}$, affecting the choice for the factorisation scale that one should use to describe $t$-channel production. An analogous argument could be made also for the shower scale choice [15], which in the MADGRAPH5_AMC@NLO matching procedure is chosen to be of the order of the partonic centre-of-mass energy in the Born process. In Fig. 8, we study the dependence of jet rates on the flavour scheme as well as on the shower scale, where two different choices of the shower scale are compared: one is the default value, and another is the default value divided by a factor of four. We can see that reducing the parton-shower scale has only a minor impact on the distributions, while a more interesting pattern arises from the choice of the flavour scheme.

For the $b$-tagged jets (right panel in Fig. 8), differences between the two schemes are rather mild $(\sim 15 \%$ in the 2-jet bin and less for 0 and 1 jet) and always compatible within the scale uncertainty, which for the 2-jet bin is much larger in the $5 \mathrm{~F}$ (the accuracy being only at $\mathrm{LO}$ ).

For non- $b$-tagged jets (left panel in Fig. 8), on the other hand, a higher jet multiplicity is clearly observed in the $4 \mathrm{~F}$ scheme, which implies that harder QCD radiation is favoured in this scheme. Interestingly, the difference is visible already at the 1-jet bin, which is described at NLO accuracy at the matrix-element level. These differences cannot arise from the small component of forward, non-taggable heavy jets; on the contrary, they can be understood by considering jets that come from genuinely light QCD radiation. In Fig. 9 we show explicitly the multiplicity of light jets only (tagged by using Monte Carlo information), both at fixed order in QCD and at NLO matched to parton shower. Our first observation is that results in the $4 \mathrm{~F}$ and $5 \mathrm{~F}$ are almost identical at fixed LO (where only the zero and one jet bins are filled). The difference is therefore an effect of higher-order corrections, as it is confirmed by observing the fixed-NLO histograms. We recall that the fixed-order matrix element has a different colour structure in different schemes; in particular, the $4 \mathrm{~F}$ at LO features a gluon in the initial state (compared to the $b$ quark in the $5 \mathrm{~F}$ ) and an extra $b$ in the final state. The radiation of extra light QCD partons from the $g \rightarrow b \bar{b}$ splitting is therefore favoured in the $4 \mathrm{~F}$ (e.g. an extra gluon can either attach to the initial-state gluon or to one of the $b$ 's, while in the $5 \mathrm{~F}$ it can attach only to the initial-state $b$ ). This is indeed what we observe at fixed NLO.

If the origin of the difference in the jet rates can be traced back to the difference between the $\mathrm{LO} 4 \mathrm{~F}$ and $5 \mathrm{~F}$ colour structures, then one would also expect this difference to be mitigated once higher-order corrections are included. To this aim, we have performed a fixed-order computation of the 2 -jet bin in the $5 \mathrm{~F}$ at $\mathrm{NLO}$ accuracy, i.e. calculated $t H j j$ at NLO, within our simulation framework, finding indeed that the rate is significantly enhanced (by $\sim 60 \%$ ), lying much closer to the $4 \mathrm{~F}$ result. A further hint that the scheme difference is indeed mitigated at higher orders is given by the $\mathrm{NLO}+\mathrm{PS}$ results, which show that the 2-jet bin in the 4F is reduced by $\sim 10 \%$ after the shower, while the corresponding $5 \mathrm{~F}$ one is enhanced by $\sim 30 \%$ over the fixed-order result. Finally, we have checked that the same results we have found here for single top plus Higgs, occur also in the case of single top production alone. In conclusion, our results suggest that the inclusion of the $g \rightarrow b \bar{b}$ splitting in the matrix-element description at the lowest order, i.e. the $4 \mathrm{~F}$ scheme, allows a wider range of observables relevant for the analyses to be described more accurately.

\section{$4 s$-channel production}

Higgs-top quark associated production at hadron colliders can also be mediated by $s$-channel diagrams, see Fig. 2. Compared to $t$-channel production, the $s$-channel mechanism is 
Fig. 9 Jet rates only for the light jets both at fixed order and matched to a parton shower in $4 \mathrm{~F}$ and $5 \mathrm{~F}$ schemes with different choices of the shower scales

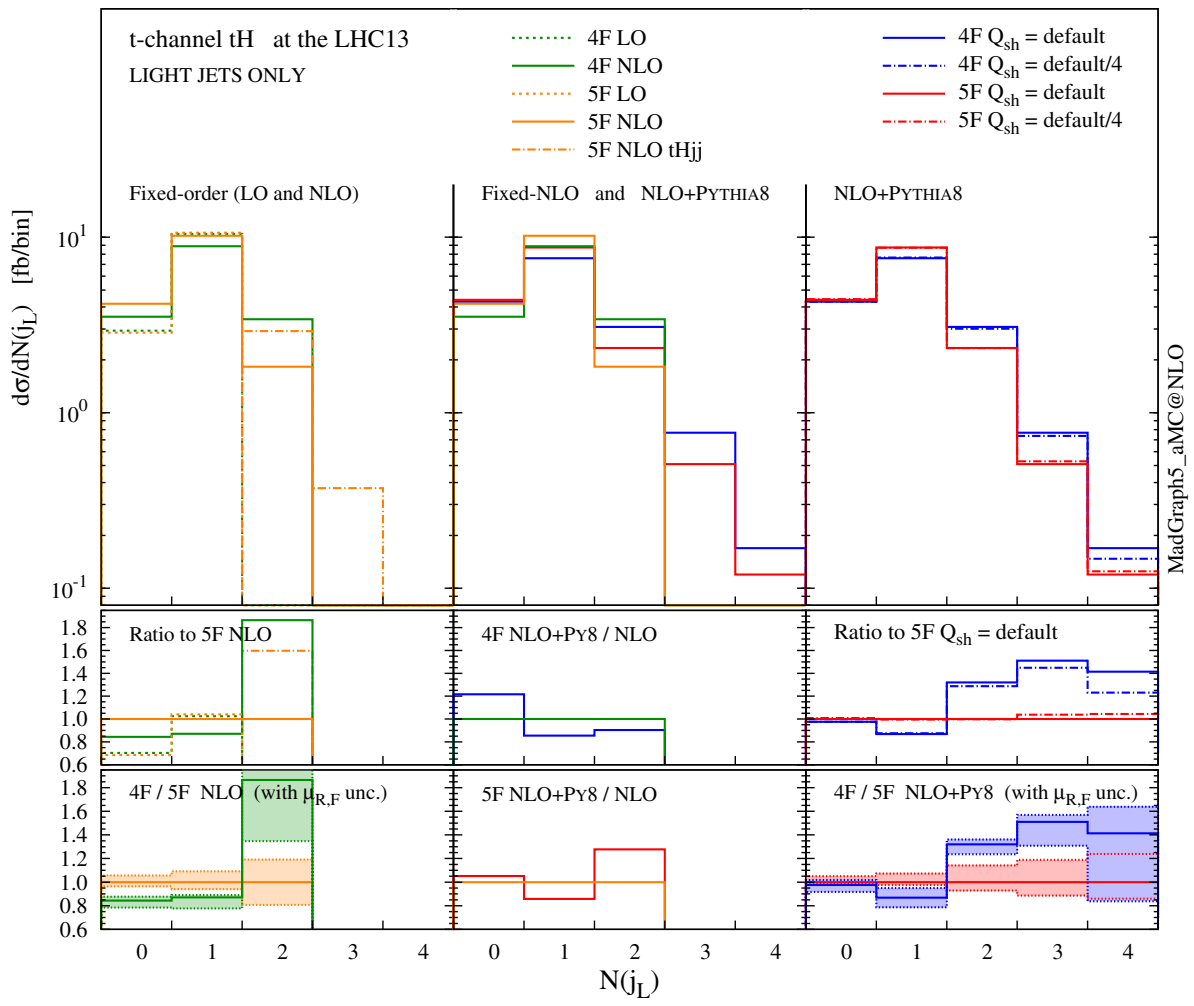

Table 5 NLO total cross section for the processes $p p \rightarrow t H \bar{b}+\bar{t} H b$ via an $s$-channel $W$-boson exchange at the $\operatorname{LHC}(\sqrt{s}=13 \mathrm{TeV})$. NNPDF2.3 PDFs have been used. The integration uncertainty in the

\begin{tabular}{lllll}
\hline$s$-channel & $\sigma_{\mathrm{NLO}}[\mathrm{fb}]$ & $\delta_{\mu}^{\%}$ & $\delta_{\mathrm{PDF}}^{\%}$ & $\delta_{\alpha_{s}}^{\%}$ \\
\hline$t H+\bar{t} H$ & $2.812(3)$ & ${ }_{-1.2}^{+1.6}$ & +1.4 & \\
\hline
\end{tabular}

naturally suppressed by the higher virtuality of the intermediate $W$ boson and features a much smaller cross section at the LHC. In this section we calculate the NLO cross section, evaluating the corresponding uncertainties, and compare $s$ channel distributions to those of $t$-channel production at NLO + PS level.

At LO, $s$-channel production proceeds through $q \bar{q}$ annihilation into a virtual $W$ boson, which can either emit a Higgs boson and then split to a $t b$ final state, or first split to $t b$ with the subsequent emission of a Higgs from the top quark. It turns out that in this case the interference between these two diagrams is positive and its effect are much less relevant than in $t$-channel production [10]. At NLO, extra radiation can take place from either initial or final state, with no interference between the two due to colour conservation. For the same reason, no interference between the $s$-channel and $t$ channel processes is present in the $5 \mathrm{~F}$ scheme and the separation between channels is still exact at NLO accuracy. In this production mode, bottom quarks are directly produced in the hard scattering via electroweak interaction and appear only in the final state. Thus, at variance with the $t$-channel last digit (in parentheses), the fractional scale dependence and the PDF and $\alpha_{s}$ uncertainties (in \%) are also reported

and $W$-associated production, the flavour scheme is not a key source of uncertainties for $s$-channel production.

In the MADGRAPH5_AMC@NLO framework the code and the events for $s$-channel production at hadron colliders can be automatically generated by typing the following commands:

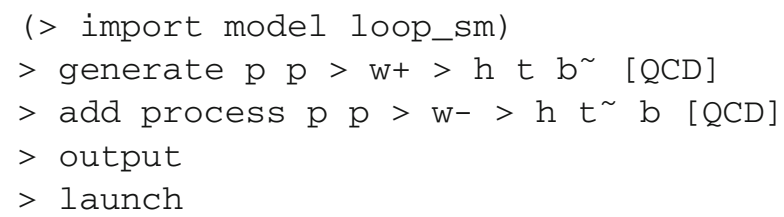

In Table 5 we show the total cross section at NLO. Reference values for the factorisation and renormalisation scales are set to $\mu_{0}=H_{T} / 2=\sum m_{\mathrm{T}} / 2$. Being a pure EW process at LO, $s$-channel production exhibits very low scale and $\alpha_{s}$ uncertainties up to NLO. In the SM, the total rate amounts to about $3 \mathrm{fb}$, i.e. less than $5 \%$ of the $t$-channel cross section.

In Figs. 10 and 11 we compare the shape of some distributions between the $s$-channel and $t$-channel production modes at NLO + PS accuracy. We can see that most of the observ- 

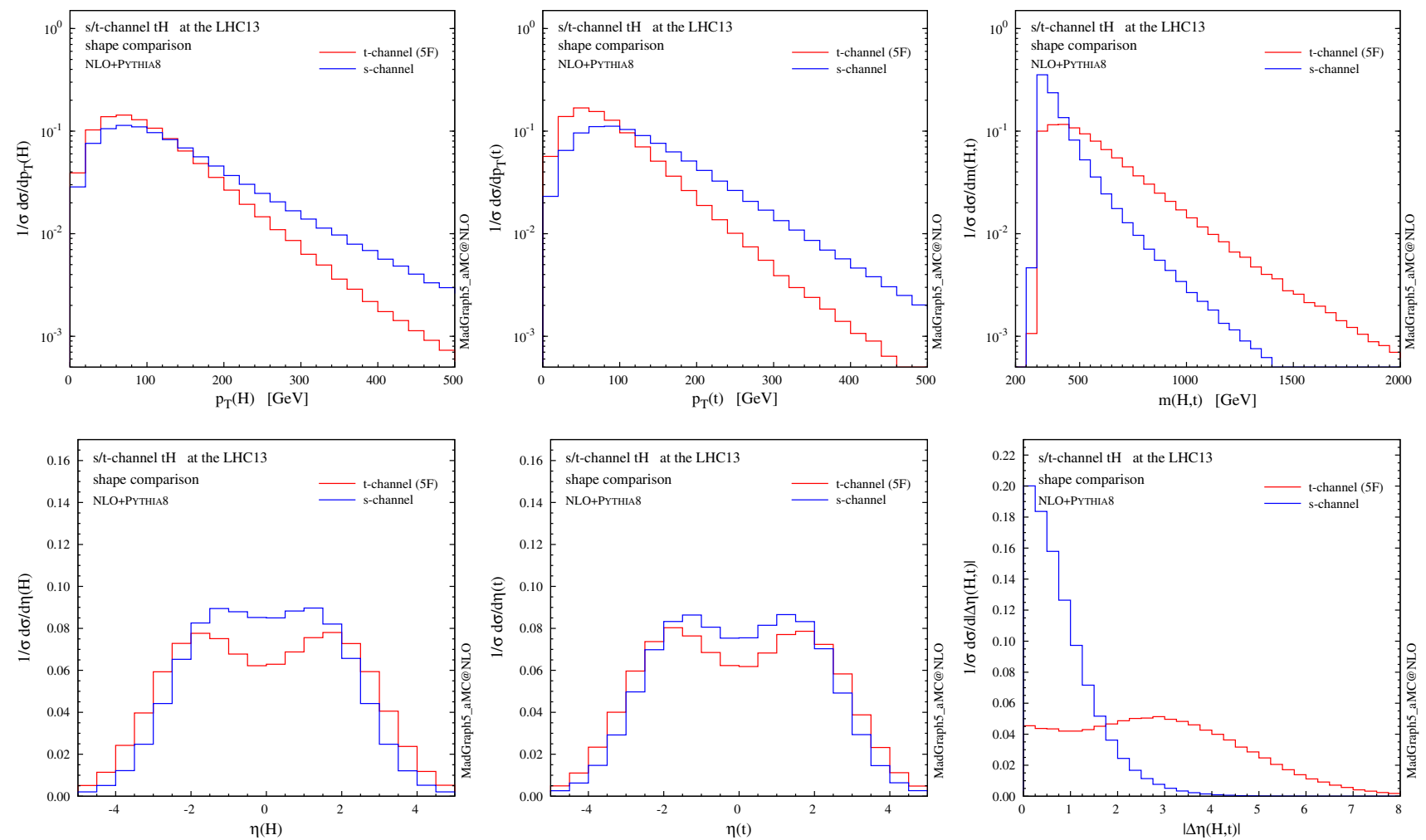

Fig. 10 Shape comparison between $s$ - and $t$-channel distributions for the Higgs boson and the top quark at NLO + PS accuracy

ables related to $s$-channel events display a significantly different shape. Even though the total cross section in $s$-channel production is tiny and deviations from a $t$-channel-only simulation would probably fall inside the uncertainty band, the $s$-channel simulation can be included with little extra computing cost when precision is needed (it is also extremely fast at NLO).

\section{Higgs characterisation}

In this section we go beyond the SM and explore the sensitivity of Higgs-single-top associated production to a Higgs boson coupling to the top quark that does not conserve CP. Several phenomenological studies on anomalous Higgs coupling determination via Higgs-single-top associated production have appeared $[8,9,51-56]$. Current experimental constraints on the Higgs-boson couplings favour the SM, and in particular for the top quark the magnitude is consistent with the SM expectations, even though an opposite sign with respect to the SM one is not yet completely excluded $[57,58]$.

Moreover, although the scenario of a pseudoscalar Higgs is disfavoured [59,60], no stringent constraint has been put on a CP-violating $H t \bar{t}$ coupling. In fact, even if current results are fully compatible with the SM hypothesis, some analyses on public LHC data seem to favour a non-zero phase in the top quark Yukawa interaction [61-64].
In this work we consider the (simplified) case of a spin0 particle with a general CP-violating Yukawa interaction with the top quark, which couples both to scalar and pseudoscalar fermionic densities. On the other hand, we assume the interaction with the $W$ bosons to be the SM one. We note that this assumption does not correspond to a typical realisation of CP-violation in a two-Higgs-doublet model where the mass eigenstates are $\mathrm{CP}$-mixed states and their coupling to the vector bosons is reduced. Our setup, however, corresponds to considering the effective SM Lagrangian and to including the operator

$\mathcal{L}=\frac{c_{t}}{\Lambda^{2}}\left(\phi^{\dagger} \phi\right) Q_{L} \tilde{\phi} t_{R}+$ h.c.

with $c_{t}$ complex. The implementation we use is based on the effective field theory framework presented in Refs. [24-26] and employs the HC_NLO_X0 model [27]. ${ }^{2}$ The effective Lagrangian for the Higgs-top quark interaction (12) below the EWSB scale leads to (see Eq. (2.2) in Ref. [24])

$\mathcal{L}_{0}^{t}=-\bar{\psi}_{t}\left(c_{\alpha} \kappa_{H t t} g_{H t t}+i s_{\alpha} \kappa_{A t t} g_{A t t} \gamma_{5}\right) \psi_{t} X_{0}$,

${ }^{2}$ For the code and event generation, one can simply issue the command 'import model $\mathrm{HC} \_\mathrm{NLO} \times \mathrm{X} 0$ ' and replace ' $\mathrm{h}$ ' by ' $\mathrm{x} 0$ ' in MADGRAPH5_AMC@NLO. 

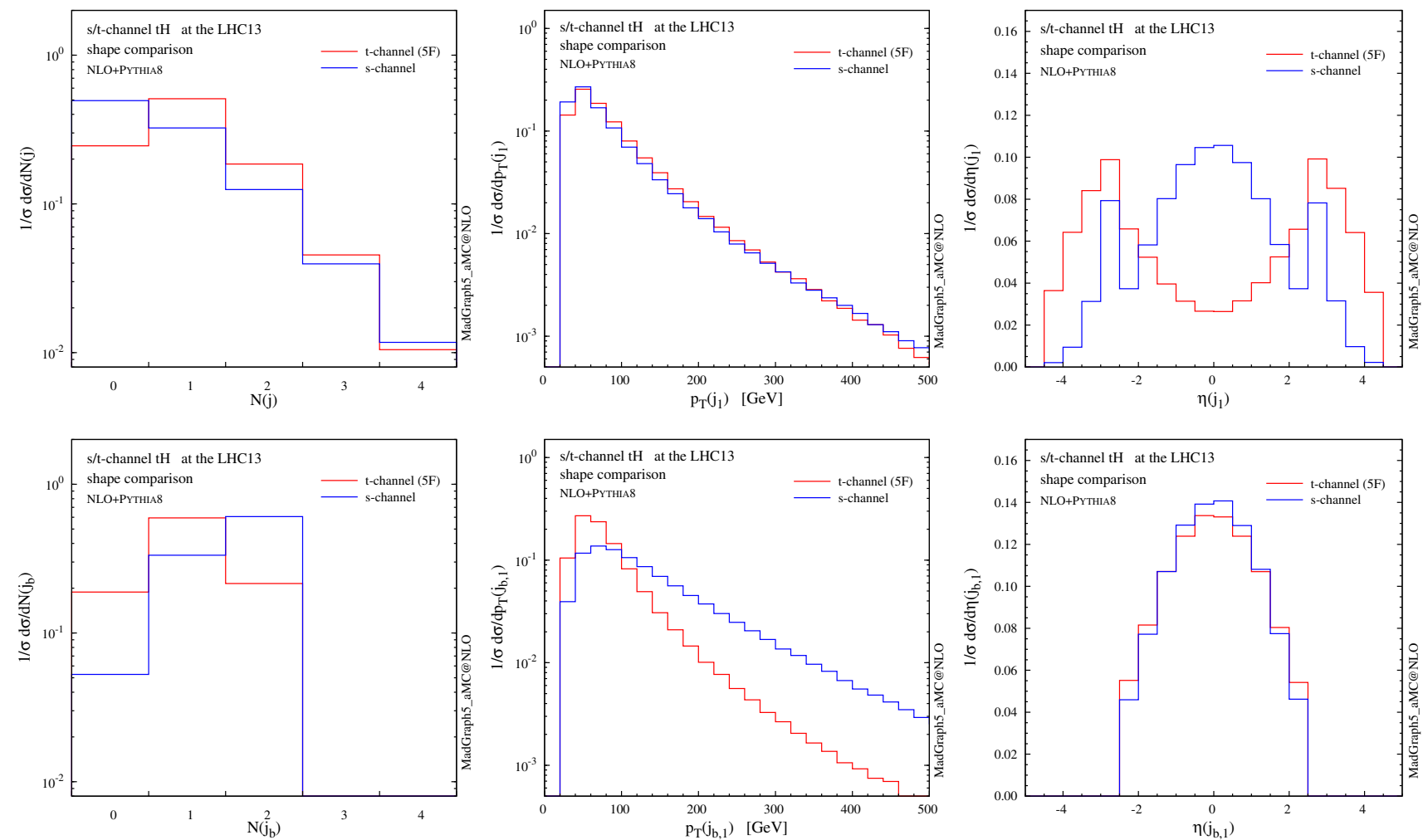

Fig. 11 In the top row shape comparison between $s$ - and $t$-channel distributions for jet rates (left), $p_{T}$ (centre) and $\eta$ (right) spectra for the hardest jet at NLO + PS accuracy. In the bottom row corresponding plots for $b$-tagged jets

where $X_{0}$ labels a generic spin-0 particle with CP-violating couplings, $c_{\alpha} \equiv \cos \alpha$ and $s_{\alpha} \equiv \sin \alpha$ are related to the CP-mixing phase $\alpha, \kappa_{H t t, A t t}$ are real dimensionless rescaling parameters, and $g_{H t t}=g_{A t t}=m_{\mathrm{t}} / v\left(=y_{t} / \sqrt{2}\right)$, with $v \sim 246 \mathrm{GeV}$. While redundant (only two independent real quantities are needed to parametrise the most general CPviolating interaction with the top quark at dimension four), this parametrisation has the practical advantage of easily interpolating between the CP-even $\left(c_{\alpha}=1, s_{\alpha}=0\right)$ and CP-odd ( $\left.c_{\alpha}=0, s_{\alpha}=1\right)$ couplings, as well as to easily recover the SM case by setting $c_{\alpha}=1, \kappa_{H t t}=1$.

The nature of the top quark Yukawa coupling directly affects the loop-induced Higgs coupling to gluons (together with an effect on the couplings to $\gamma \gamma$ and $Z \gamma$, which are also modified but not considered here)

$\mathcal{L}_{0}^{g}=-\frac{1}{4}\left(c_{\alpha} \kappa_{H g g} g_{H g g} G_{\mu \nu}^{a} G^{a, \mu \nu}+s_{\alpha} \kappa_{A g g} g_{A g g} G_{\mu \nu}^{a} \widetilde{G}^{a, \mu \nu}\right) X_{0}$,

where $g_{H g g}=-\alpha_{S} /(3 \pi v)$ and $g_{A g g}=\alpha_{S} /(2 \pi v)$. In the parametrisation given above, the strength of the coupling between Higgs and gluons can be rescaled independently of the top quark Yukawa coupling. Assuming that the the top quark dominates the gluon-fusion (GF) process at the LHC energies, then $\kappa_{H g g} \rightarrow \kappa_{H t t}, \kappa_{A g g} \rightarrow \kappa_{A t t}$. In so doing, the ratio between the actual cross section for GF at NLO QCD and the corresponding SM prediction can be written as

$\frac{\sigma_{\mathrm{NLO}}^{g g \rightarrow X_{0}}}{\sigma_{\mathrm{NLO}, \mathrm{SM}}^{g g \rightarrow H}}=c_{\alpha}^{2} \kappa_{H t t}^{2}+s_{\alpha}^{2}\left(\kappa_{A t t} \frac{g_{A g g}}{g_{H g g}}\right)^{2}$,

because there is no interference between the scalar and pseudoscalar components in the amplitudes for Higgs plus up to three external partons, see e.g., [26]. In particular, if the rescaling parameters are set to

$\kappa_{H t t}=1, \quad \kappa_{A t t}=\left|g_{H g g} / g_{A g g}\right|=2 / 3$,

the SM GF cross section is reproduced for every value of the CP-mixing phase $\alpha$. Given that current measurements are compatible with the expected SM GF production rate, one can consider the simplified scenario where the condition in Eq. (16) is imposed and the CP-mixing phase $\alpha$ is basically left unconstrained by current data.

Figure 12 shows the total cross section for $t$-channel $t X_{0}$ production as a function of the CP-mixing angle $\alpha$. We also show the $t \bar{t} X_{0}$ cross section, which is not only another process sensitive to the modifications of the top quark Yukawa coupling in Eq. (13), but also a background to $t$-channel production. The uncertainty band represents the envelope 


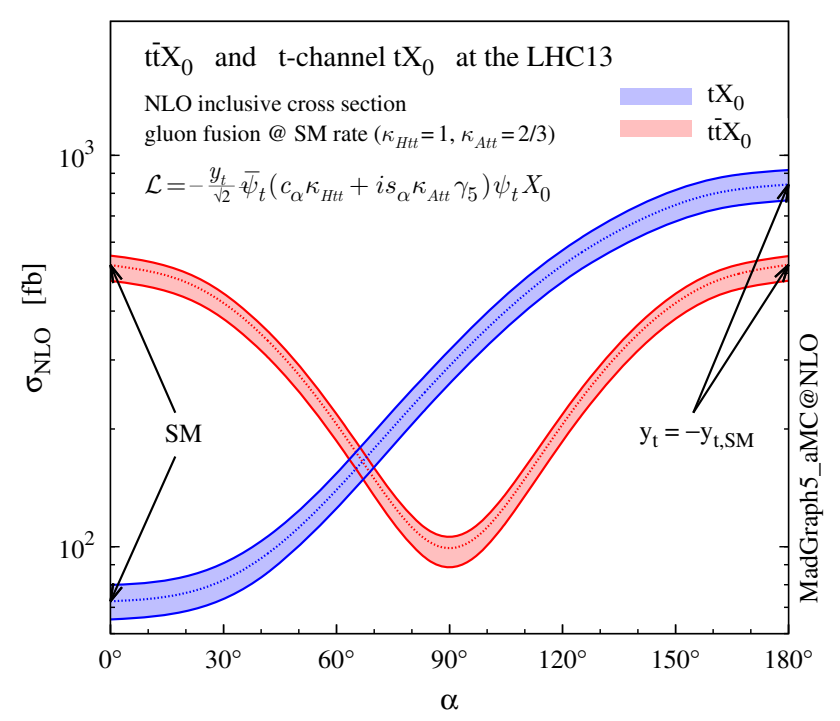

Fig. 12 NLO cross sections (with scale uncertainties) for $t \bar{t} X_{0}$ and $t$-channel $t X_{0}$ productions at the $13-\mathrm{TeV}$ LHC as a function of the CPmixing angle $\alpha$, where $\kappa_{H t t}$ and $\kappa_{A t t}$ are set to reproduce the SM GF cross section for every value of $\alpha$

defined in Sect. 3.2, i.e. the combined scale and flavourscheme dependence. The $t \bar{t} X_{0}$ uncertainty band represents the scale dependence only, when the scale is varied by a factor two around $\mu_{0}=\sqrt[3]{m_{\mathrm{T}}(t) m_{\mathrm{T}}(\bar{t}) m_{\mathrm{T}}\left(X_{0}\right)}$ [26].

The first important observation is that while the GF and $t \bar{t} H$ cross sections are degenerate under $y_{t} \rightarrow-y_{t}$ (depending quadratically from the top quark Yukawa coupling), in $t$-channel production this degeneracy is clearly lifted by the interference between diagrams where the Higgs couples to the top quark and to the $W$ boson. In $[8,9]$ it was shown that the $t$-channel cross section is enhanced by more than one order of magnitude when the strength of the top Yukawa coupling is changed in sign with respect to the SM value. Here we can see how the same enhancement can take place also in the presence a continuous rotation in the scalar-pseudoscalar plane. While not affecting GF (by construction), such a rotation has an impact also on the $t \bar{t} X_{0}$ rate, which is in general lower for a pseudoscalar or CP-mixed state [26]. $t$-channel production lifts another degeneracy present in GF and $t \bar{t} X_{0}$, namely $\alpha \rightarrow \pi-\alpha$. Given the partial compensation between the $t$-channel and $t \bar{t} X_{0}$ cross sections at different values of $\alpha$, an analysis which could well separate between the two production mechanisms would be needed to put stringent constraints on a CP-violating Higgs coupling to the top quark.

We remind that the enhancement of the $t$-channel cross section takes place mostly at threshold, as one can clearly see in the left plot of Fig. 13. This means that one should not be concerned by violations of perturbative unitarity at the LHC, as they do not appear for partonic centre-of-mass energies lower than $\sim 10 \mathrm{TeV}$ [9]. In Fig. 13 we also show the transverse momentum distributions for the Higgs and the top quark. The distributions are well behaved in this case too, not displaying any strong trend in their high- $p_{T}$ tails, i.e anything that could suggest a unitarity violating behaviour.

Finally, in Fig. 14 we plot the pseudorapidity separation between the Higgs and the top quark (left) and the opening angle between the hardest jet and the lepton from the top quark in the lab frame (right), showing that these variables have a discriminating power on $\alpha$. For this last observable, the lepton is required to satisfy the following selection criteria

$p_{T}(\ell)>20 \mathrm{GeV}, \quad|\eta(\ell)|<2.5$.

\section{Summary}

In this work we have studied the production of a Higgs boson in association with a single top quark at the LHC. Our aim has been to carefully consider the effects of NLO corrections in QCD on total cross sections and differential distributions for $t$ - and $s$-channel production. We have scrutinised a wide range of theoretical systematic uncertainties and in particu-
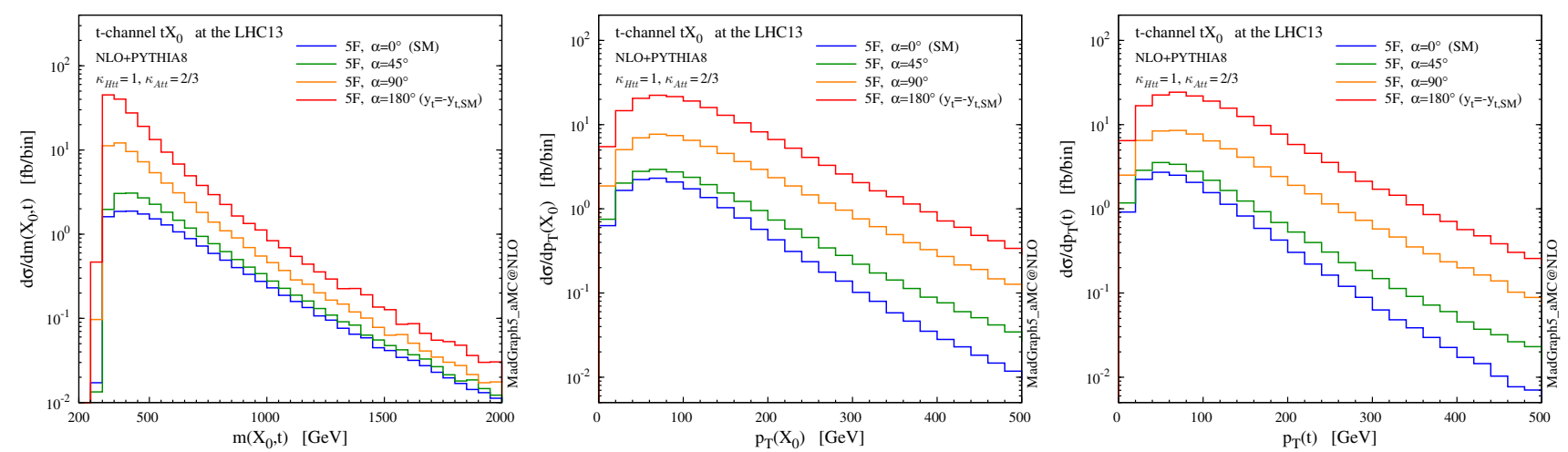

Fig. 13 Differential distributions for the Higgs boson and the top quark at NLO + PS accuracy in $t$-channel $t H$ associated production at the 13-TeV LHC, with different values of the CP-mixing angles, where $\kappa_{H t t}$ and $\kappa_{A t t}$ are set in Eq. (16) to reproduce the SM GF cross section for every value of $\alpha$ 


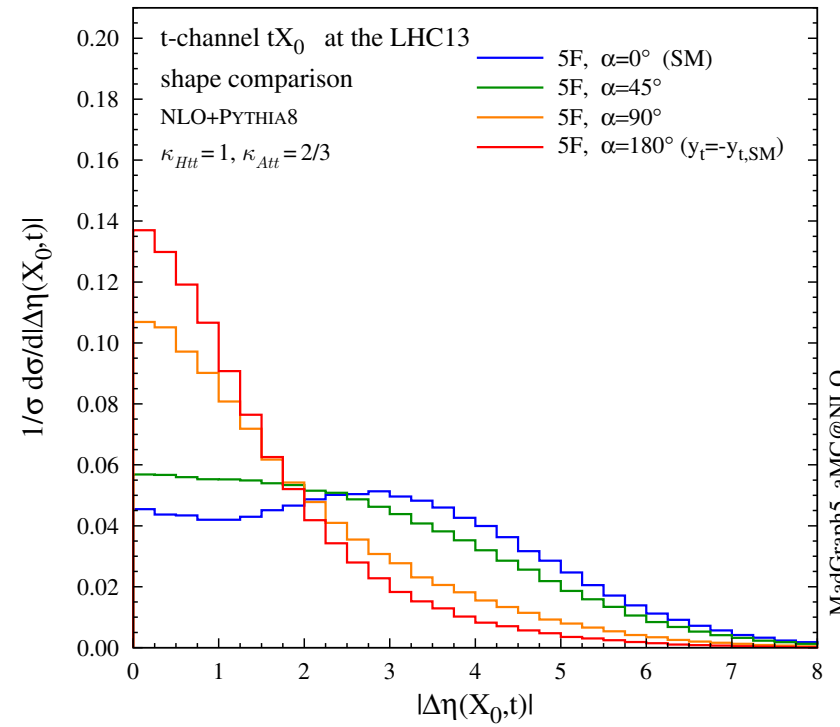

Fig. 14 Shape comparison among different values of the CP-mixing angles, where $\kappa_{H t t}$ and $\kappa_{A t t}$ are set in Eq. (16) to reproduce the SM GF cross section for every value of $\alpha$. Pseudorapidity separation between

lar those arising from the choice of the heavy-quark scheme, 4-flavour or 5-flavour. We have found that at the level of total cross sections a comfortable consistency between the two schemes exists when physically motivated choices for the renormalisation and factorisation scales are made, with similar resulting uncertainties. For differential distributions, on the other hand, the situation is slightly more involved. While sizeable differences between the two schemes arise at LO, they are considerably milder at NLO and NLO + PS, in line with expectations. In this case, we have shown that the $4 \mathrm{~F}$ and $5 \mathrm{~F}$ schemes provide fully consistent and similarly precise predictions for distributions such as those of the Higgs boson, the top quark, and the forward jet. On the other hand, the 4-flavour scheme is in general able to provide accurate predictions for a wider set of observables, including those of the spectator $b$-quark and extra jets. In addition to $t$-channel production in the SM, we have also briefly presented the results for the subdominant $s$-channel production, highlighting the differences in the most important distributions with respect to the corresponding ones of $t$-channel production. Finally, we have provided results (total cross sections as well as a few representative distributions) for the case where an explicit $\mathrm{CP}$ violation is present in the coupling between the top quark and the Higgs boson, making it clear that in this case Higgs associated production with a single top could provide complementary and very valuable information to that of $t \bar{t} H$ production. We conclude by stressing that all results presented here have been obtained by employing the publicly available MADGRAPH5_AMC@NLO framework and therefore they can be easily reproduced (and possibly extended) by generating

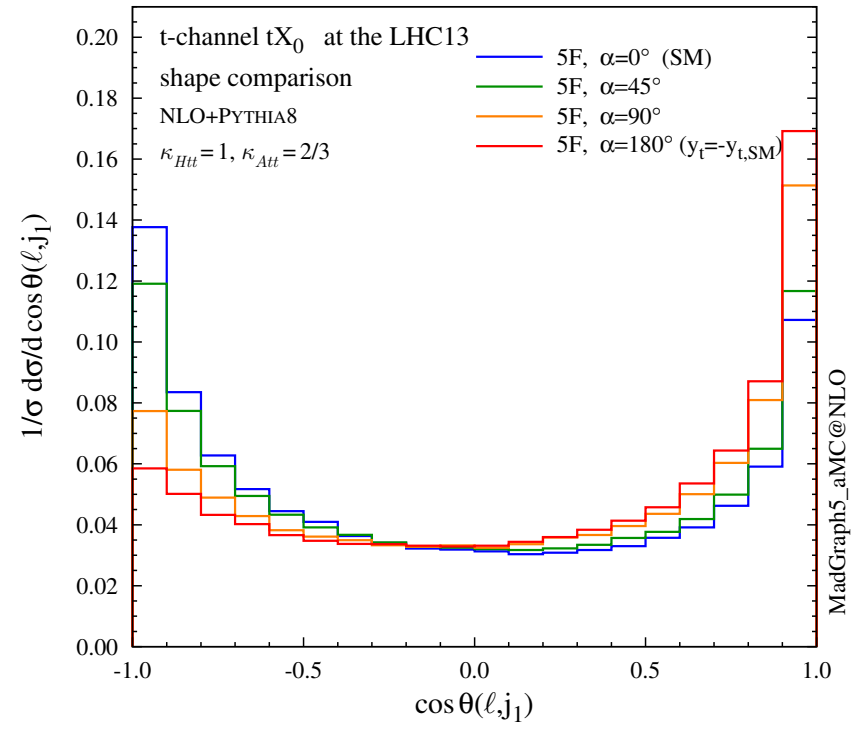

the Higgs and the top quark (left $)$ and opening angle between the hardest jet and the lepton from the top quark in the lab frame (right)

the corresponding event samples to be used in fully-fledged experimental analyses.

Acknowledgments We thank the LHC HXSWG and in particular the members of the $t t H / t H j$ task force for giving us the motivation to pursue this study and for many stimulating discussions. We are grateful to Rikkert Frederix and Stefano Frixione for discussions and to Paolo Torrielli for his valuable help. This work has been performed in the framework of the ERC Grant 291377 "LHCtheory: Theoretical predictions and analyses of LHC physics: advancing the precision frontier" and of the FP7 Marie Curie Initial Training Network MCnetITN (PITN-GA-2012-315877). It is also supported in part by the Belgian Federal Science Policy Office through the Interuniversity Attraction Pole P7/37. The work of FD and FM is supported by the IISN "MadGraph" convention 4.4511.10 and the IISN "Fundamental interactions" convention 4.4517.08. KM is supported in part by the Strategic Research Program "High Energy Physics" and the Research Council of the Vrije Universiteit Brussel. The work of MZ is supported by the ERC grant "Higgs@LHC" and partially by the ILP LABEX (ANR-10-LABX-63), in turn supported by French state funds managed by the ANR within the "Investissements d'Avenir" programme under Reference ANR-11IDEX-0004-02.

Open Access This article is distributed under the terms of the Creative Commons Attribution 4.0 International License (http://creativecomm ons.org/licenses/by/4.0/), which permits unrestricted use, distribution, and reproduction in any medium, provided you give appropriate credit to the original author(s) and the source, provide a link to the Creative Commons license, and indicate if changes were made. Funded by SCOAP ${ }^{3}$.

\section{References}

1. F. Englert, R. Brout, Broken symmetry and the mass of gauge vector mesons. Phys. Rev. Lett. 13, 321-323 (1964) 
2. P.W. Higgs, Broken symmetries and the masses of gauge bosons. Phys. Rev. Lett. 13, 508-509 (1964)

3. S. Weinberg, Implications of dynamical symmetry breaking. Phys. Rev. D 13, 974-996 (1976)

4. CMS-HIG-13-003. CMS-HIG-13-004. CMS-HIG-13-006. CMSHIG-13-009 (2013)

5. ATLAS-CONF-2013-009. ATLAS-CONF-2013-010. ATLASCONF-2013-012. ATLAS- CONF-2013-013 (2013)

6. W. Buchmuller, D. Wyler, Effective Lagrangian analysis of new interactions and flavor conservation. Nucl. Phys. B 268, 621 (1986)

7. B. Grzadkowski, M. Iskrzynski, M. Misiak, J. Rosiek, Dimensionsix terms in the standard model Lagrangian. JHEP 1010, 085 (2010). arXiv: 1008.4884

8. S. Biswas, E. Gabrielli, B. Mele, Single top and Higgs associated production as a probe of the Htt coupling sign at the LHC. JHEP 1301, 088 (2013). arXiv:1211.0499

9. M. Farina, C. Grojean, F. Maltoni, E. Salvioni, A. Thamm, Lifting degeneracies in Higgs couplings using single top production in association with a Higgs boson. JHEP 1305, 022 (2013). arXiv: 1211.3736

10. F. Maltoni, K. Paul, T. Stelzer, S. Willenbrock, Associated production of Higgs and single top at hadron colliders. Phys. Rev. D 64, 094023 (2001). arXiv:hep-ph/0106293

11. M. Kramer, F.I. Olness, D.E. Soper, Treatment of heavy quarks in deeply inelastic scattering. Phys. Rev. D 62, 096007 (2000). arXiv:hep-ph/0003035

12. M. Brucherseifer, F. Caola, K. Melnikov, On the NNLO QCD corrections to single-top production at the LHC. Phys. Lett. B 736, 58-63 (2014). arXiv:1404.7116

13. J.M. Campbell, R. Frederix, F. Maltoni, F. Tramontano, Nextto-leading-order predictions for t-channel single-top production at hadron colliders. Phys. Rev. Lett. 102, 182003 (2009). arXiv:0903.0005

14. F. Maltoni, G. Ridolfi, M. Ubiali, $b$-initiated processes at the LHC: a reappraisal. JHEP 1207, 022 (2012). arXiv:1203.6393

15. M. Wiesemann, R. Frederix, S. Frixione, V. Hirschi, F. Maltoni et al., Higgs production in association with bottom quarks. JHEP 1502, 132 (2015). arXiv: 1409.5301

16. J. Alwall, R. Frederix, S. Frixione, V. Hirschi, F. Maltoni et al., The automated computation of tree-level and next-to-leading order differential cross sections, and their matching to parton shower simulations. JHEP 1407, 079 (2014). arXiv: 1405.0301

17. S. Frixione, B.R. Webber, Matching NLO QCD computations and parton shower simulations. JHEP 0206, 029 (2002). arXiv:hep-ph/0204244

18. G. Ossola, C.G. Papadopoulos, R. Pittau, CutTools: a program implementing the OPP reduction method to compute one-loop amplitudes. JHEP 0803, 042 (2008). arXiv:0711.3596

19. R. Frederix, S. Frixione, F. Maltoni, T. Stelzer, Automation of nextto-leading order computations in QCD: the FKS subtraction. JHEP 10, 003 (2009). arXiv:0908.4272

20. V. Hirschi et al., Automation of one-loop QCD corrections. JHEP 05, 044 (2011). arXiv: 1103.0621

21. C. Degrande, C. Duhr, B. Fuks, D. Grellscheid, O. Mattelaer et al., UFO - the Universal FeynRules Output. Comput. Phys. Commun. 183, 1201-1214 (2012). arXiv:1108.2040

22. A. Alloul, N.D. Christensen, C. Degrande, C. Duhr, B. Fuks, FeynRules 2.0-a complete toolbox for tree-level phenomenology. Comput. Phys. Commun. 185, 2250-2300 (2014). arXiv:1310.1921

23. P. de Aquino, W. Link, F. Maltoni, O. Mattelaer, T. Stelzer, ALOHA: automatic libraries of helicity amplitudes for Feynman diagram computations. Comput. Phys. Commun. 183, 2254-2263 (2012). arXiv: 1108.2041

24. P. Artoisenet, P. de Aquino, F. Demartin, R. Frederix, S. Frixione et al., A framework for Higgs characterisation. JHEP 1311, 043 (2013). arXiv:1306.6464
25. F. Maltoni, K. Mawatari, M. Zaro, Higgs characterisation via vector-boson fusion and associated production: NLO and partonshower effects, Eur. Phys. J. C 74(1), 2710 (2014). arXiv:1311.1829

26. F. Demartin, F. Maltoni, K. Mawatari, B. Page, M. Zaro, Higgs characterisation at NLO in QCD: CP properties of the topquark Yukawa interaction. Eur. Phys. J. C 74(9), 3065 (2014). arXiv:1407.5089

27. http://feynrules.irmp.ucl.ac.be/wiki/HiggsCharacterisation

28. J. Alwall, A. Ballestrero, P. Bartalini, S. Belov, E. Boos et al., A standard format for Les Houches event files. Comput. Phys. Commun. 176, 300-304 (2007). arXiv:hep-ph/0609017

29. P. Artoisenet, R. Frederix, O. Mattelaer, R. Rietkerk, Automatic spin-entangled decays of heavy resonances in Monte Carlo simulations. JHEP 1303, 015 (2013). arXiv: 1212.3460

30. S. Frixione, E. Laenen, P. Motylinski, B.R. Webber, Angular correlations of lepton pairs from vector boson and top quark decays in Monte Carlo simulations. JHEP 0704, 081 (2007). arXiv:hep-ph/0702198

31. A. Martin, W. Stirling, R. Thorne, G. Watt, Heavy-quark mass dependence in global PDF analyses and 3- and 4-flavour parton distributions. Eur. Phys. J. C 70, 51-72 (2010). arXiv:1007.2624

32. R.D. Ball, V. Bertone, S. Carrazza, C.S. Deans, L. Del Debbio et al., Parton distributions with LHC data. Nucl. Phys. B 867, 244-289 (2013). arXiv:1207.1303

33. A. Martin, W. Stirling, R. Thorne, G. Watt, Parton distributions for the LHC. Eur. Phys. J. C 63, 189-285 (2009). arXiv:0901.0002

34. H.-L. Lai, M. Guzzi, J. Huston, Z. Li, P.M. Nadolsky et al., New parton distributions for collider physics. Phys. Rev. D 82, 074024 (2010). arXiv:1007.2241

35. M. Whalley, D. Bourilkov, R. Group, The Les Houches accord PDFs (LHAPDF) and LHAGLUE. arXiv:hep-ph/0508110

36. S. Alekhin, S. Alioli, R. D. Ball, V. Bertone, J. Blumlein, et al., The PDF4LHC working group interim report. arXiv:1101.0536

37. M. Botje, J. Butterworth, A. Cooper-Sarkar, A. de Roeck, J. Feltesse, et al., The PDF4LHC working group interim recommendations. arXiv: 1101.0538

38. Particle Data Group Collaboration, K. Olive et al., Review of particle physics. Chin. Phys. C 38, 090001 (2014)

39. A. Martin, W. Stirling, R. Thorne, G. Watt, Uncertainties on alpha(S) in global PDF analyses and implications for predicted hadronic cross sections. Eur. Phys. J. C 64, 653-680 (2009). arXiv:0905.3531

40. H.-L. Lai, J. Huston, Z. Li, P. Nadolsky, J. Pumplin et al., Uncertainty induced by QCD coupling in the CTEQ global analysis of parton distributions. Phys. Rev. D 82, 054021 (2010). arXiv: 1004.4624

41. S. Lionetti, R.D. Ball, V. Bertone, F. Cerutti, L. Del Debbio et al., Precision determination of $\alpha_{s}$ using an unbiased global NLO parton set. Phys. Lett. B 701, 346-352 (2011). arXiv:1103.2369

42. T. Sjostrand, S. Mrenna, P.Z. Skands, A. Brief, Introduction to PYTHIA 8.1. Comput. Phys. Commun. 178, 852-867 (2008). arXiv:0710.3820

43. G. Corcella, I. Knowles, G. Marchesini, S. Moretti, K. Odagiri et al., HERWIG 6: an event generator for hadron emission reactions with interfering gluons (including supersymmetric processes). JHEP 0101, 010 (2001). arXiv:hep-ph/0011363

44. T. Sjostrand, S. Mrenna, P.Z. Skands, PYTHIA 6.4 physics and manual. JHEP 0605, 026 (2006). arXiv:hep-ph/0603175

45. M. Bahr, S. Gieseke, M. Gigg, D. Grellscheid, K. Hamilton et al., Herwig++ physics and manual. Eur. Phys. J. C 58, 639-707 (2008). arXiv:0803.0883

46. M. Cacciari, G.P. Salam, G. Soyez, The anti-k(t) jet clustering algorithm. JHEP 0804, 063 (2008). arXiv:0802.1189

47. M. Cacciari, G.P. Salam, G. Soyez, FastJet user manual. Eur. Phys. J. C 72, 1896 (2012). arXiv:1111.6097 
48. R.D. Ball, S. Carrazza, L. Del Debbio, S. Forte, J. Gao et al., Parton distribution benchmarking with LHC data. JHEP 1304, 125 (2013). arXiv: 1211.5142

49. ATLAS, CDF, CMS, D0 Collaboration, First combination of Tevatron and LHC measurements of the top-quark mass. arXiv: 1403.4427

50. ATLAS, CMS Collaboration, G. Aad et al., Combined measurement of the Higgs boson mass in $p p$ collisions at $\sqrt{s}=7$ and 8 $\mathrm{TeV}$ with the ATLAS and CMS experiments. arXiv: 1503.0758

51. J. Yue, $\mathcal{C} \mathcal{P}$-violating top-Higgs coupling and top polarisation in the diphoton decay of the $t h j$ channel at the LHC. arXiv:1410.2701

52. A. Kobakhidze, L. Wu, J. Yue, Anomalous top-Higgs couplings and top polarisation in single top and Higgs associated production at the LHC. JHEP 1410, 100 (2014). arXiv:1406.1961

53. J. Chang, K. Cheung, J.S. Lee, C.-T. Lu, Probing the top-Yukawa coupling in associated Higgs production with a single top quark. JHEP 1405, 062 (2014). arXiv: 1403.2053

54. C. Englert, E. Re, Bounding the top Yukawa coupling with Higgsassociated single-top production, Phys. Rev. D 89(7), 073020 (2014). arXiv:1402.0445

55. J. Ellis, D.S. Hwang, K. Sakurai, M. Takeuchi, Disentangling Higgs-top couplings in associated production. JHEP 1404, 004 (2014). arXiv:1312.5736

56. P. Agrawal, S. Mitra, A. Shivaji, Effect of anomalous couplings on the associated production of a single top quark and a Higgs boson at the LHC. JHEP 1312, 077 (2013). arXiv:1211.4362
57. CMS Collaboration, V. Khachatryan et al., Precise determination of the mass of the Higgs boson and tests of compatibility of its couplings with the Standard Model predictions using proton collisions at 7 and $8 \mathrm{TeV}$. arXiv: 1412.8662

58. ATLAS Collaboration, G. Aad et al., Updated coupling measurements of the Higgs boson with the ATLAS detector using up to 25 $\mathrm{fb}^{-1}$ of proton-proton collision data. ATLAS-CONF-2014-009

59. ATLAS Collaboration, Study of the spin of the new boson with up to $25 \mathrm{fb}^{-1}$ of ATLAS data. ATLAS-CONF-2013-040

60. CMS Collaboration, V. Khachatryan et al., Constraints on the spinparity and anomalous HVV couplings of the Higgs boson in proton collisions at 7 and $8 \mathrm{TeV}$. arXiv: 1411.3441

61. A. Djouadi, G. Moreau, The couplings of the Higgs boson and its $\mathrm{CP}$ properties from fits of the signal strengths and their ratios at the $7+8$ TeV LHC. Eur. Phys. J. C 73, 2512 (2013). arXiv:1303.6591

62. K. Cheung, J.S. Lee, P.-Y. Tseng, Higgs precision (Higgcision) era begins. JHEP 1305, 134 (2013). arXiv:1302.3794

63. K. Nishiwaki, S. Niyogi, A. Shivaji, tt $H$ anomalous coupling in double Higgs production. JHEP 1404, 011 (2014). arXiv: 1309.6907

64. F. Boudjema, R.M. Godbole, D. Guadagnoli, K.A. Mohan, Lab-frame observables for probing the top-Higgs interaction. arXiv: 1501.03157 